\title{
Convergence Analysis of Saddle Point Problems in Time Varying Wireless Systems - Control Theoretical Approach
}

\author{
Junting Chen and Vincent K. N. Lau
}

\begin{abstract}
Saddle point problems arise from many wireless applications, and primal-dual iterative algorithms are widely applied to find the saddle points. In the existing literature, the convergence results of such algorithms are established assuming the problem specific parameters remain unchanged during the iterations. However, this assumption is unrealistic in time varying wireless systems, as explicit message passing is usually involved in the iterations and the channel state information (CSI) may change in a time scale comparable to the algorithm update period. This paper investigates the convergence behavior and the tracking error of primal-dual iterative algorithms under time varying CSI. The convergence results are established by studying the stability of an equivalent virtual dynamic system derived in the paper, and the Lyapunov theory is applied for the stability analysis. We show that the average tracking error is proportional to the time variation rate of the CSI. Based on these analyses, we also derive an adaptive primal-dual algorithm by introducing a compensation term to reduce the tracking error under the time varying CSI.
\end{abstract}

\section{Index Terms}

Saddle Point, Convex Optimization, Convergence Analysis, Time-Varying, Lyapunov Stability, Network Utility Maximization

\section{INTRODUCTION}

Saddle point problems arise in a number of wireless communication applications such as resource allocations and competitive games. Resource allocations problems can be formulated as a constrained maximization of some utility functions. By constructing a Lagrangian function, the constrained problem can be reformulated into an unconstrained one and be solved by computing the saddle point of the Lagrangian function, where we maximize over the primal variables and minimize over the dual variables. Most remarkably, primal-dual gradient methods have been widely used for computing the saddle points of general Lagrangian functions, while they provide decentralized solutions in wireless applications. The primal-dual gradient methods update the primal and dual variables simultaneously by evaluating the gradient of both the primal function and dual function at the same time. A classical study of primal-dual algorithms has been done by Arrow, Hurwicz and Uzawa in their seminal work [1], where they constructed a continuous-time algorithm for general concave-convex functions and derived the asymptotic properties using Lyapunov-like methods. They also provided a global stability 
result on a discrete-time algorithm with constant step sizes. Recently, Feijer et al. [2], [3] have studied the stability of these primal-dual algorithm dynamics and extended the results to various network resource allocations problems.

In the above literature, when people discuss the convergence behavior of the primal-dual gradient algorithm, all the problem specific parameters are considered to be time invariant. Even in most other literatures studying optimization problems in wireless communications, problem dependent parameters are merely considered to be quasi-static, meaning that these time varying parameters are assumed to be constant for a sufficiently long time until the algorithms converge. However, this assumption is unrealistic, especially in wireless communication scenarios. For instance, the operating environment in terms of the channel state information (CSI) may be changing frequently, such that the optimization problem varies from time to time. On the other hand, as explicit message passing may be involved in the iterations, the optimization algorithms cannot always converge fast enough to catch up with time varying effects, especially for large scale problems. As is shown in our numerical example, convergence errors would lead to performance loss in a wireless communication system. However, it is yet unknown whether the algorithms converge or not when the quasi-static assumption is dropped, even for strictly convex problems. Therefore, it is highly important to the study the convergence behavior, or robustness, for primal-dual gradient algorithms under time varying CSI.

However, towards this end there are a lot of technical challenges, some of which are listed below.

- How to quantify the performance penalty due to the time varying parameters

- How to evaluate the cost-performance tradeoff

- How to enhance the algorithm

These questions are highly nontrivial due to the stochastic nature of wireless communication problems as well as the complexities of the algorithms that solve them. There are some preliminary works studying the effects caused by time varying parameters. For example, for resource allocation problems, the authors in [4] had introduced stochastic perturbations to the algorithm to represent random loads entering the network, and their analyses were based on linearization on the system. The authors in [5] studied a stochastic network utility maximization (NUM) problem with noisy feedback input to the algorithm. However, [4] and [5] did not consider the CSI being time varying and hence their problems have static equilibrium points. In [6], the authors studied the performance of gradient algorithms for a game problem with moving equilibria, and the authors in [7], [8] studied multi-cell CDMA interference games under time varying channels. While these work [6], [7], [8] provide some preliminary results for solving games under time varying CSI, their techniques cannot be applied to general saddle point problems.

In this paper, we shall investigate the convergence behavior of primal-dual algorithms for solving general saddle point problems under time varying CSI. We first define an equivalent virtual dynamic 
system, following which we study the stability of such virtual dynamic system based on the Lyapunov theory [9] and the LaSalle's invariance principle argument [10]. We model the dynamics of the time varying CSI as an auto-regressive system, and the stability results are derived by constructing Lyapunov functions along the algorithm trajectories. Based on the stability results, we derive the convergence properties of primal-dual algorithms. We also quantify the average tracking errors in terms of the average exogenous excitations induced to the CSI dynamics. Furthermore, to enhance the tracking performance, we propose a novel adaptive algorithm with distributive implementation solutions. As an illustration, we also consider two wireless application examples, based on which we show that the numerical results match with our analysis.

The paper is organized as follows. In Section II] we introduce a general saddle point problem as well as the primal-dual algorithm with two application examples in wireless communication. We also introduce the time varying CSI model, and the virtual dynamic systems. Then in Section III and Section [V], we analyze the convergence behavior of the primal-dual algorithm for the strongly concave-convex saddle point problem and degraded saddle point problem, respectively. In Section $\mathrm{V}$ we propose a novel adaptive algorithm to reduce the tracking error in time varying CSI. Section VI gives the simulation results and discussions. Finally, we summarize our main results in Section VII

Notations: Matrices and vectors are denoted by capitalized and lowercase boldface letters, respectively. $A^{T}\left(\mathbf{a}^{T}\right)$ denotes the transpose of matrices (vectors) $A$ (a), and $A^{H}$ denotes the complex conjugate transpose. $\mathbb{R}$ and $\mathbb{C}$ denote the set of real numbers and complex numbers, respectively. $\mathbf{a} \succeq \mathbf{b}$ denotes the entry-by-entry comparison and $A \succeq B$ means that $A-B$ is positive semidefinite. Unless specified in the context, $\|\cdot\|$ denotes the Euclidean norm.

\section{SyStem Model And ViRTUAL DynAmic Systems}

In this section, we shall first introduce a general saddle point problem setup and illustrate with a few examples in the context of wireless communications. We shall then define the time varying CSI model and discuss its impact on the convergence of saddle point problems. Finally, we shall introduce the notion of virtual dynamic system and establish the equivalence between convergence behavior of the saddle point algorithm and the stability of the virtual dynamic system.

\section{A. General Saddle Point Problem}

We consider a min-max optimization problem

$$
\min _{\lambda \in \mathbb{R}_{+}^{m}} \max _{\mathbf{x} \in \mathbb{R}^{n}} \mathcal{L}(\mathbf{x}, \lambda ; \mathbf{h})
$$

where the function $\mathcal{L}: \mathbb{R}^{n} \times \mathbb{R}_{+}^{m} \mapsto \mathbb{R}$ has two vector variables. Moreover, $\mathcal{L}(\mathbf{x}, \lambda ; \mathbf{h})$ is strongly concave in $\mathbf{x} \in \mathbb{R}^{n}$ and convex in $\lambda \in \mathbb{R}_{+}^{m} . \mathbf{h} \in \mathcal{H} \subseteq \mathbb{R}^{q}$ is a vector parameter that arises from 
specific optimization problems. In the context of wireless communication optimizations, the problem parameter $\mathbf{h}$ can be the CSI. Under the above convexity assumption, the min-max problem has a unique optimal solution $\left(\mathbf{x}^{*}, \lambda^{*}\right)$. Note that since the min-max problem in (1) is parameterized by $\mathbf{h}$, the optimal solution $\left(\mathbf{x}^{*}, \lambda^{*}\right)$ can also be represented as a mapping from $\mathbf{h} \in \mathcal{H}$ to $\left(\mathbf{x}^{*}, \lambda^{*}\right)$

$$
\mathbf{x}^{*}(\mathbf{h})=\psi_{x}(\mathbf{h}), \lambda^{*}(\mathbf{h})=\psi_{\lambda}(\mathbf{h}) \quad \forall \mathbf{h} \in \mathcal{H}
$$

where $\psi_{x}: \mathcal{H} \mapsto \mathbb{R}^{n}$ and $\psi_{\lambda}: \mathcal{H} \mapsto \mathbb{R}_{+}^{m}$ are $\mathcal{C}^{1}$ functions.

It is known that solving the above min-max optimization problem (1) is equivalent to computing the saddle point of $\mathcal{L}(\mathbf{x}, \lambda ; \mathbf{h})[11]$. For a given $\mathbf{h} \in \mathcal{H}$, a saddle point $\left(\mathbf{x}^{*}, \lambda^{*}\right)$ of $\mathcal{L}(\mathbf{x}, \lambda ; \mathbf{h})$ over the set $\mathbb{R}^{n} \times \mathbb{R}_{+}^{m}$ is defined to be a vector that satisfies

$$
\mathcal{L}\left(\mathbf{x}, \lambda^{*} ; \mathbf{h}\right) \leq \mathcal{L}\left(\mathbf{x}^{*}, \lambda^{*} ; \mathbf{h}\right) \leq \mathcal{L}\left(\mathbf{x}^{*}, \lambda ; \mathbf{h}\right), \quad \forall \mathbf{x} \in \mathbb{R}^{n}, \lambda \in \mathbb{R}_{+}^{m}
$$

It is also known that when $\mathcal{L}$ is strongly concave in $\mathrm{x}$ and convex in $\lambda$, the saddle point is unique [11], meaning that the optimal solution to (1) is unique.

The classical primal-dual gradient algorithm dynamics ${ }^{1}$ for solving (1) are given in the following:

$$
\begin{aligned}
& \dot{\mathbf{x}}=\frac{d \mathbf{x}}{d t}=\kappa\left[\frac{\partial}{\partial \mathbf{x}} \mathcal{L}(\mathbf{x}, \lambda ; \mathbf{h}(t))\right]^{T} \\
& \dot{\lambda}=\frac{d \lambda}{d t}=\kappa\left[\left(-\frac{\partial}{\partial \lambda} \mathcal{L}(\mathbf{x}, \lambda ; \mathbf{h}(t))\right)^{T}\right]_{\lambda}^{+}
\end{aligned}
$$

for some step size $\kappa>0$. The projection []$_{\lambda}^{+}$is to restrict $\lambda$ in the nonnegative domain $\mathbb{R}_{+}^{m}$. For scalars $u_{i}$ and $\lambda_{i}$, the projection is defined to be $\left[u_{i}\right]_{\lambda_{i}}^{+}:=u_{i}$ if $u_{i}>0$ or $\lambda_{i}>0$, and $\left[u_{i}\right]_{\lambda_{i}}^{+}:=0$ otherwise. For the vector case, the projection is defined entry-wide. This algorithm was first introduced by Arrow et al. [1] and has been recently studied and applied to various resource allocations applications [3]. The asymptotic behavior has been analyzed by studying the Lyapunov function of the system state and applying LaSalle's invariant principle argument [9], [10].

In all these works, the convergence results were established based on the assumption that the wireless channel state $\mathbf{h}$ stays time invariant before the algorithm converges. In practice, the iterations in (2)-(3) may involve explicit message passing among nodes in a wireless network, and hence it is quite unlikely for the $\mathbf{h}$ to be invariant during the iterations. When the channel state $\mathbf{h}(t)$ is time varying, all the existing convergence results [1], [3] fail to apply. In this paper, we shall address the situation where the CSI $\mathbf{h}(t)$ changes in a similar time scale as the primal-dual algorithm in (2)-(3), which reflects a more realistic situation in practice.

\footnotetext{
${ }^{1}$ In this paper, we assume the step size of iterations are sufficiently small so that the primal-dual iterations can be represented by the continuous time dynamics [1], [3].
} 


\section{B. Examples of Saddle Point Problems in Wireless Communications}

In this subsection, we shall illustrate various important applications of saddle point problems in the context of wireless communications.

1) Application 1: Transmission and Jamming Strategy Optimization in MIMO Channels: Consider a point-to-point MIMO system with a jammer as shown in Fig. 1. The transmitter and the receiver have $N$ antennas. The user $\mathrm{X}$ transmits the desired signal $\mathrm{x} \sim \mathcal{C N}(0, \mathbf{Q})$ to the receiver $\mathrm{Y}$, while the jammer $\mathrm{Z}$ transmits a jamming signal $\mathbf{z} \sim \mathcal{C N}(0, \mathbf{Z})$ to interfere $\mathrm{Y}$. The received signal at $\mathrm{Y}$ is given by $\mathbf{y}=\mathbf{H}_{1} \mathbf{x}+\mathbf{H}_{2} \mathbf{z}+\mathbf{n}$, where $\mathbf{n} \sim \mathcal{C N}\left(0, \sigma_{n}^{2} \mathbf{I}\right)$ is the additive Gaussian noise, and the mutual information between $\mathrm{X}$ and $\mathrm{Y}$ is given by [12]

$$
C(\mathbf{Q}, \mathbf{Z})=\log \operatorname{det}\left(\mathbf{I}+\left(\sigma_{n}^{2} \mathbf{I}+\mathbf{H}_{2} \mathbf{Z} \mathbf{H}_{2}^{H}\right)^{-1} \mathbf{H}_{1} \mathbf{Q} \mathbf{H}_{1}^{H}\right) .
$$

Assume that both the user and jammer have perfect transmit CSI (CSIT), and they know each other's transmission covariance. Thus the user's strategy is to maximize the mutual information given in (4) based on the CSIT and the knowledge of the jammer's transmission covariance, while the the jammer's strategy is to minimize that mutual information. Their strategies result in finding the saddle point of the following saddle point problem [13], [14].

Problem 1 (Transmission and Jamming Strategy Optimization):

$$
\min _{\operatorname{tr}(\mathbf{Z}) \leq P_{\mathcal{J}}} \max _{\operatorname{tr}(\mathbf{Q}) \leq P_{T}} C(\mathbf{Q}, \mathbf{Z} ; \mathbf{h})=\log \operatorname{det}\left(\mathbf{I}+\left(\sigma_{n}^{2} \mathbf{I}+\mathbf{H}_{2} \mathbf{Z} \mathbf{H}_{2}^{H}\right)^{-1} \mathbf{H}_{1} \mathbf{Q} \mathbf{H}_{1}^{H}\right) .
$$

where $\mathbf{h}=\operatorname{vec}\left(\left[\mathbf{H}_{1} \mathbf{H}_{2}\right]\right), P_{T}$ and $P_{\mathcal{J}}$ are the total power constraints for the user and jammer, respectively.

Note that the saddle point problem in (5) is strongly concave in $\mathbf{Q}$ and strongly convex in $\mathbf{Z}$ [14], and hence there exists a unique saddle point, which can be computed by a primal-dual iterative algorithm (2)-(3). When the CSI $\mathbf{h}(t)$ is time invariant, the transmission policies for both the user and jammer will converge to the saddle point of (5), and a Nash Equilibrium is achieved. However, when the CSI $\mathbf{h}(t)$ is time varying in a similar timescale as the primal-dual iterations, the convergence is not guaranteed. Hence, it is interesting to study the convergence behavior of the algorithm iterations under time-varying CSI.

2) Application 2: Network Utility Maximization of Wireless Ad Hoc Network: In a resource allocation problem, the communication network is modeled as a set of source nodes transmitting traffic through a set of links. Each source node has its own concave utility function and is allocated a portion of resources. The optimization problem is to maximize the total network utility under a set of constraints on the total network resources. The problem is formulated as follows [4], [15]. 
Problem 2 (Network Utility Maximization):

$$
\begin{array}{cc}
\max _{\mathbf{r} \succeq 0, \mathbf{p} \succeq 0} & \sum_{(s, d) \in \mathcal{C}} U_{s d}\left(r_{s d}\right) \\
\text { subject to } & \sum_{(s, d): l \in L(s, d)} r_{s d} \leq c_{l}\left(p_{l} ; \mathbf{h}\right) \quad \forall l \\
\mathbf{p} \in \mathcal{P} &
\end{array}
$$

where the ordered pair $(s, d)$ denotes the traffic, each of which has a fixed route, that initiates from source node $s$ and is delivered to destination node $d, r_{s d}$ denotes the data rate for the traffic, $\mathbf{p}=\left[p_{1} \ldots p_{L}\right]^{T}$ is a vector of power allocated to $L$ links, $U_{s d}$ is the utility function to evaluate traffic rate $r_{s d}$ for source $s$, and $c_{l}(\cdot)$ is a link capacity function of the transmit power $p_{l}$ and channel gain (i.e. CSI) h. $\mathcal{C}$ denotes the collection of all traffic flows, $L(s, d)$ denotes the set of links that traffic $(s, d)$ goes through, and $\mathcal{P}$ denotes the set of feasible power vectors. We assume both $U_{s d}(\cdot)$ and $c_{l}(\cdot)$ are strongly concave and twice differentiable. Fig. 2 2 illustrates an example NUM problem with 3 nodes, 2 links, and $|\mathcal{C}|=3$ traffic flows. For example, the collection of traffic flows is given by $\mathcal{C}=\{(1,2),(1,3),(2,3)\}$, and the sets of links are $L(1,2)=\{1\}, L(1,3)=\{1,2\}$ and $L(2,3)=\{2\}$.

For notation convenience, we denote $\mathbf{x}=[\mathbf{r} ; \mathbf{p}]$ as a vector of the primal variables. The Lagrangian of (6) can be written as

$$
\mathcal{L}(\mathbf{r}, \mathbf{p}, \lambda ; \mathbf{h})=\sum_{s \in \mathcal{S}} U\left(r_{s}\right)-\sum_{l} \lambda_{l}\left(\sum_{s: l \in L(s)} r_{s}-c_{l}\left(p_{l} ; \mathbf{h}\right)\right)
$$

subjected to the power constraint $\mathbf{p} \in \mathcal{P}$. From the Lagrangian theory [11], solving the optimization problem (6) is equivalent to finding the saddle point of the Lagrangian function (7),

$$
\min _{\lambda \succeq 0} \max _{\mathbf{r} \succeq 0 ; \mathbf{p} \in \mathcal{P}} \mathcal{L}(\mathbf{r}, \mathbf{p}, \lambda ; \mathbf{h})
$$

which is a special instance of (1), and the primal-dual algorithm (2)-(3) can be applied to solve this problem.

Note that as solving the dual problem involves explicit message passing among nodes, the convergence rate of the algorithm is critical to the solution quality of the NUM problem. Since it may not be justified in practice that the channel state $\mathbf{h}(t)$ is time invariant during the algorithm convergence, it is very important to study the convergence behavior of the primal-dual iteration in (2)-(3) when the CSI is time varying.

\section{Time Varying CSI Model}

In this section, we first introduce a dynamic model for the time-varying CSI $\mathbf{h}(t)$. Specifically, the CSI $\mathbf{h}(t)$ is modeled as a solution to the following dynamic equation

$$
\dot{\mathbf{h}}(t)=\frac{d \mathbf{h}(t)}{d t}=A(\mathbf{h}(t)-\overline{\mathbf{h}})+u(t)
$$


where $A$ is a real symmetric negative definite matrix, $u(t)$ is a vector valued complex Gaussian process with uncorrelated real and imaginary components, and $\overline{\mathbf{h}}$ is a constant vector, corresponding to the line-of-sight (LOS) component in the channel model. The dynamic system can be viewed as an external disturbance $u(t)$ being applied to an autonomous $\mathrm{s}^{2}$ linear system $\dot{\mathbf{h}}=A(\mathbf{h}(t)-\overline{\mathbf{h}})$. Note that the dynamic model 9 resembles an $\mathrm{AR}(1)$ process and $|\mathbf{h}(t)|$ has a stationary Rician distribution. As is summarized in [16], the AR models have been successfully used to model, predict and simulate fading channel dynamics (e.g. in [17], [16], [18]), the autocorrelation function of which was also observed and justified in [19] to be close to that of a Rayleigh fading process.

Note that the dynamic system model of $\mathbf{h}$ in (9) is stable $]^{3}[9]$. Firstly, if $u(t)=0$, the dynamic system (9) exponentially converges to the unique equilibrium point $\mathbf{h}=\overline{\mathbf{h}}$. Secondly, when $u(t) \neq 0$, it is also stable provided that $\overline{\|u(t)\|^{2}}=\lim _{T \rightarrow \infty} \frac{1}{T} \int_{0}^{T}\|u(t)\|^{2} d t<\infty$. This can be verified by constructing a Lyapunov function $L(\mathbf{h})=(\mathbf{h}-\overline{\mathbf{h}})^{T}(\mathbf{h}-\overline{\mathbf{h}}) / 2$ on the state $\mathbf{h}$ and applying the LaSalle's invariant principle argument [10], [9]. The stability property in the CSI model $\mathbf{h}(t)$ is important because it is a necessary condition for the convergence of the primal-dual algorithm (2)(3).

\section{Virtual Dynamic Systems}

In this section, we shall first define a virtual dynamic system. We shall illustrate that studying the convergence behavior of the primal-dual iterative algorithm for the saddle point problem in (2)-(3) is the same as studying the stability behavior of the virtual dynamic system. As a result, the virtual dynamic system forms a bridge between the convergence analysis of iterative algorithms and nonlinear control theory.

Definition 1 (Virtual Dynamic System): Let $\widetilde{\mathbf{x}}=(\mathbf{x}, \lambda)$ be the joint state of the primal and dual variables in algorithm (2)-(3) and let $\tilde{f}: \mathbb{R}^{n} \times \mathbb{R}_{+}^{m} \mapsto \mathbb{R}^{n+m}$ be the vector valued function

$$
\widetilde{f}(\widetilde{\mathbf{x}} ; \mathbf{h}(t)) \triangleq\left[\begin{array}{c}
\kappa\left(\frac{\partial}{\partial \mathbf{x}} \mathcal{L}(\mathbf{x}, \lambda ; \mathbf{h}(t))\right)^{T} \\
\kappa\left[\left(-\frac{\partial}{\partial \lambda} \mathcal{L}(\mathbf{x}, \lambda ; \mathbf{h}(t))\right)^{T}\right]_{\lambda}^{+}
\end{array}\right] .
$$

The state $\widetilde{\mathbf{x}}$ of the virtual dynamic system satisfies the following dynamic equation

$$
\widetilde{\mathcal{X}}: \quad \dot{\widetilde{\mathbf{x}}}=\frac{d}{d t} \widetilde{\mathbf{x}}(t)=\widetilde{f}(\widetilde{\mathbf{x}} ; \mathbf{h}(t)) .
$$

Definition 2 (Equilibrium Point): $\widetilde{\mathbf{x}}^{*}$ is called an equilibrium point of the vector field $\dot{\widetilde{\mathbf{x}}}=\widetilde{f}(\widetilde{\mathbf{x}} ; \mathbf{h}(t))$, if $\widetilde{f}\left(\widetilde{\mathbf{x}}^{*} ; \mathbf{h}(t)\right)=0$.

\footnotetext{
${ }^{2}$ An autonomous system is a system that can be described by an ordinary differential equation $\dot{x}=f(x)$ where $f$ does not explicitly depend on $t$ [9].

${ }^{3}$ The concept of stability is formally introduced in Definition 4 in the following subsection.
} 
Note that since the vector field $\dot{\widetilde{\mathbf{x}}}=\widetilde{f}(\widetilde{\mathbf{x}} ; \mathbf{h})$ is parameterized by the channel state $\mathbf{h}(t)$, the equilibrium point $\widetilde{\mathbf{x}}^{*}(\mathbf{h}(t))$ is expressed as a function of $\mathbf{h}(t)$. From (11) and Definition 2, we have the following result establishing the connection between the virtual dynamic system (11) and the primal-dual algorithm (2)-(3) for solving saddle point problems.

Theorem 1 (Connections between the Virtual Dynamic System and the Saddle Point Problem): The equilibrium point $\widetilde{\mathbf{x}}^{*}(\mathbf{h}(t))$ of the virtual dynamic system (11) is identical to the saddle point $\left[\mathbf{x}^{*}(\mathbf{h}(t)), \lambda^{*}(\mathbf{h}(t))\right]$ of the saddle point problem (1). Furthermore, the algorithm trajectories of the primal-dual algorithm in (2)-(3) are the same as those of the virtual dynamic system (11).

Proof: Please refer to Appendix A for the proof.

As a result of Theorem 1, the convergence behavior of the primal-dual algorithm (2)-(3) can be visualized by the stability of the equilibrium point in the virtual dynamic system (11). For instance, the algorithm in (2)-(3) converges to the saddle point if and only if the equilibrium point of the virtual system (11) is asymptotically stable. When the channel state $\mathbf{h}$ is time invariant, the equilibrium point $\widetilde{\mathbf{x}}^{*}(\mathbf{h})$ is stationary and the algorithm trajectory of the primal-dual iterations can be visualized to converge to the stationary equilibrium point. On the other hand, when the channel state $\mathbf{h}(t)$ is time varying (with comparable time scale as the algorithm trajectory), the equilibrium point $\widetilde{\mathbf{x}}^{*}(\mathbf{h}(t)$ ) will be time varying and the convergence of the algorithm cannot be guaranteed. This is illustrated in Fig. 3. Due to this association, we shall focus on studying the stability of the moving equilibrium $\widetilde{\mathbf{x}}^{*}(\mathbf{h}(t))$ of the virtual system (11) in the rest of the paper.

Due to the time varying CSI, the equilibrium point $\widetilde{\mathbf{x}}^{*}(\mathbf{h}(t))$ is non-stationary. For mathematical convenience, we consider a change of state variable. Define $\widetilde{\mathbf{x}}_{e}(t)=\widetilde{\mathbf{x}}(t)-\widetilde{\mathbf{x}}^{*}(t)$. Note that $\widetilde{\mathbf{x}}_{e}(t)$ represents the instantaneous error $t$, Let $\psi(\mathbf{h}):=\left(\mathbf{x}^{*}(\mathbf{h}), \lambda^{*}(\mathbf{h})\right)=\widetilde{\mathbf{x}}^{*}(\mathbf{h})$ be a mapping from the CSI $\mathbf{h} \in \mathcal{H}$ to the equilibrium point of the virtual dynamic system (11). As $\dot{\widetilde{\mathbf{x}}}_{e}=\dot{\widetilde{\mathbf{x}}}-\dot{\widetilde{\mathbf{x}}}^{*}$, The error process $\widetilde{\mathbf{x}}_{e}(t)$ satisfies the dynamic equation of the error dynamic system defined below.

Definition 3 (Error Dynamic System): The error dynamic system for the virtual system $\dot{\widetilde{\mathbf{x}}}=\widetilde{f}(\widetilde{\mathbf{x}} ; \mathbf{h}(t))$ is given by

$$
\widetilde{\mathcal{X}}_{e}: \quad \dot{\widetilde{\mathbf{x}}}_{e}=\widetilde{f}_{e}\left(\widetilde{\mathbf{x}}_{e} ; \mathbf{h}(t)\right)-\varphi(\mathbf{h}) \dot{\mathbf{h}}(t)
$$

where $\widetilde{f}_{e}\left(\widetilde{\mathbf{x}}_{e} ; \mathbf{h}(t)\right):=\widetilde{f}\left(\widetilde{\mathbf{x}}_{e}+\psi(\mathbf{h}) ; \mathbf{h}(t)\right)$ and $\varphi(\mathbf{h}):=\frac{\partial}{\partial \mathbf{h}} \psi(\mathbf{h})$.

Since the error state $\widetilde{\mathbf{x}}_{e}(t)$ and the virtual system state $\widetilde{\mathbf{x}}(t)$ are related simply by a linear transformation, studying the stability of $\widetilde{\mathcal{X}}$ is equivalent to studying the stability of $\widetilde{\mathcal{X}}_{e}$. Finally, we summarize the formal definition of as follows [9].

\footnotetext{
${ }^{4}$ Using Theorem 11 $\widetilde{\mathbf{x}}_{e}(t)$ also represents the error between the current solution $(\mathbf{x}(t), \lambda(t))$ driven by the iterative algorithm in (2)-33 and the instantaneous saddle point $\left(\mathbf{x}^{*}(t), \lambda^{*}(t)\right)$.
} 
Definition 4 (Exponential Stability of the Virtual Dynamic System): The equilibrium point $\widetilde{\mathbf{x}}_{e}^{*}=0$ of (12) is exponentially stable if there exist some positive constants $c, k$, and $\lambda$ such that

$$
\left\|\widetilde{\mathbf{x}}_{e}(t)\right\| \leq k\left\|\widetilde{\mathbf{x}}_{e}\left(t_{0}\right)\right\| e^{-\lambda\left(t-t_{0}\right)}, \forall\left\|\widetilde{\mathbf{x}}_{e}\left(t_{0}\right)\right\|<c .
$$

It is globally exponentially stable if $(13)$ is satisfied for any initial state $\widetilde{\mathbf{x}}_{e}\left(t_{0}\right)$.

Using Theorem 1, we have global asymptotic stability of the virtual dynamic system corresponding to the global asymptotic convergence of the primal-dual algorithm in the original saddle point problem and vice versa.

\section{Convergence Analysis of Strongly Concave-Convex Saddle Point Problems UNDER TIME VARYING CSI}

In this section, we shall focus on the convergence analysis of strongly concave-convex saddle point problems when the CSI is time varying. In this case, for the saddle point function $\mathcal{L}$, the Hessian $\nabla_{x}^{2} \mathcal{L} \preceq-M_{x} \mathbf{I}$ for $M_{x}>0$ is negative definite and $\nabla_{\lambda}^{2} \mathcal{L} \succeq M_{\lambda} \mathbf{I}$ for $M_{\lambda}>0$ is positive definite. We shall first establish the key stability results of the virtual dynamic system under a virtual exogenous input. Based on that, we shall discuss the impact of time varying CSI to the convergence of the saddle point problem, followed by some examples.

\section{A. Stability Analysis of Virtual Dynamic System}

We shall study the stability of the virtual dynamic system $\widetilde{\mathcal{X}}_{e}$ in $(12)$ associated with the primal-dual iterative algorithm of a strongly concave-convex saddle point problem in (2)-(3). We first consider the case when the CSI is time invariant $(\dot{\mathbf{h}}=0)$. The following lemma summarizes the key results.

Lemma 1 (Stability of $\widetilde{\mathcal{X}}_{e}$ with Time Invariant CSI): When the CSI $\mathbf{h}(t)$ is time invariant, the equilibrium point $\widetilde{\mathbf{x}}_{e}^{*}=0$ of the virtual dynamic system $\widetilde{\mathcal{X}}_{e}$ in $(12)$ is globally exponentially stable for all $\mathbf{h} \in \mathcal{H}$.

Proof: Please refer to Appendix B for the proof.

The above convergence result is not surprising since the CSI is time invariant and similar results have been established in [1], [3], [11] using different approaches. In the following, we shall extend the stability result to the system with time varying CSI.

As an intermediate, we first consider a virtual dynamic system $\widetilde{\mathcal{X}}_{e}$ in 12 with a quasi-time varying CSI $\mathbf{h}(t)$. Specifically, $\mathbf{h}(t)$ is time varying according to the CSI model in (9) with $u(t)=0$, for all $t$. As a result, the CSI $\mathbf{h}(t)$ varies only during some transient and will converge to $\overline{\mathbf{h}}$ as $t \rightarrow \infty$. Define a joint system state as $\widetilde{\mathbf{z}}_{e}=\left(\widetilde{\mathbf{x}}_{e}, \mathbf{h}_{e}\right)$, where $\mathbf{h}_{e}=\mathbf{h}-\overline{\mathbf{h}}$, the virtual dynamic system of state $\widetilde{\mathbf{z}}_{e}$ can be specified as 


$$
\widetilde{\mathcal{Z}}_{e}: \quad \dot{\tilde{\mathbf{z}}}_{e}=\widetilde{\mathcal{Z}}\left(\widetilde{\mathbf{z}}_{e}\right):=\left[\begin{array}{c}
\widetilde{f}_{e}\left(\widetilde{\mathbf{x}}_{e} ; \mathbf{h}_{e}\right)+\varphi\left(\mathbf{h}_{e}+\overline{\mathbf{h}}\right) A \mathbf{h}_{e} \\
A \mathbf{h}_{e}
\end{array}\right] .
$$

where the $A$ is the coefficient matrix in the channel model (9). Note that $\widetilde{\mathbf{z}}_{e}^{*}=0$ is an equilibrium point for $\dot{\tilde{\mathbf{z}}}_{e}=\widetilde{\mathcal{Z}}\left(\widetilde{\mathbf{z}}_{e}\right)$. The following lemma summarizes the stability results of the virtual system $\widetilde{\mathcal{Z}}_{e}$.

Lemma 2 (Stability of $\widetilde{\mathcal{Z}}_{e}$ under Quasi-Time Varying CSI): Assume the following inequality holds for all $\mathbf{h} \in \mathcal{H}$,

$$
\|\varphi(\mathbf{h}) A\|<\kappa \min \left\{2 M,-\lambda_{\max }(A)\right\}
$$

where $\lambda_{\max }(A)$ denotes the largest eigenvalue of $A$, and $M=\min \left\{M_{x}, M_{\lambda}\right\}$. The joint virtual system $\dot{\widetilde{\mathbf{z}}}_{e}=\widetilde{\mathcal{Z}}\left(\widetilde{\mathbf{z}}_{e}\right)$ has an exponentially stable equilibrium at $\widetilde{\mathbf{z}}_{e}^{*}=0$, and there exists a Lyapunov function $V: \mathbb{R}^{n+m+q} \mapsto \mathbb{R}$ satisfying

$$
\begin{gathered}
a_{1}\left\|\widetilde{\mathbf{z}}_{e}\right\|^{2} \leq V\left(\widetilde{\mathbf{z}}_{e}\right) \leq a_{2}\left\|\widetilde{\mathbf{z}}_{e}\right\|^{2} \\
\dot{V}\left(\widetilde{\mathbf{z}}_{e}\right)=\frac{\partial V}{\partial \mathbf{z}_{e}} \widetilde{\mathcal{Z}}\left(\widetilde{\mathbf{z}}_{e}\right) \leq-a_{3}\left\|\widetilde{\mathbf{z}}_{e}\right\|^{2} \\
\left\|\frac{\partial V}{\partial \widetilde{\mathbf{z}}_{e}}\right\| \leq a_{4}\left\|\widetilde{\mathbf{z}}_{e}\right\|
\end{gathered}
$$

for some positive constants $a_{1}, a_{2}, a_{3}$ and $a_{4}>0$.

Proof: Please refer to Appendix $\mathrm{C}$ for the proof.

Remark 1 (Interpretation of Lemma 2): From Lemma 2, even though CSI $\mathbf{h}(t)$ is quasi-time varying, we still need condition (15) to guarantee globally exponential stability during the transient of $\mathbf{h}(t)$. In fact, the parameter $\kappa$ affects the convergence rate of the primal-dual algorithm, the eigenvalues of $A$ represent the the transient rate of $\mathbf{h}(t)$ and the value $\|\varphi(\mathbf{h})\|$ can be considered as the sensitivity of the moving equilibrium $\widetilde{\mathbf{x}}_{e}^{*}(t)$ to the variation of $\mathbf{h}(t)$. The result of Lemma 2 illustrates that when the transient rate of $\mathbf{h}(t)$ is slow and the sensitivity of the moving equilibrium is small, the underlying virtual dynamic system $\widetilde{\mathcal{Z}}_{e}$ still has global exponential stability. This property will be used to study the stability of the virtual system under time varying CSI.

Finally, we consider the stability result of the virtual dynamic system $\widetilde{\mathcal{Z}}_{e}$ under the time varying CSI model in (9). Specifically, the virtual dynamic system is given by

$$
\begin{aligned}
\widetilde{\mathcal{Z}}_{e}(u): \quad \dot{\widetilde{\mathbf{z}}}_{e} & =\left[\begin{array}{c}
\widetilde{f}_{e}\left(\widetilde{\mathbf{x}}_{e} ; \mathbf{h}_{e}\right)+\varphi\left(\mathbf{h}_{e}+\overline{\mathbf{h}}\right) A \mathbf{h}_{e} \\
A \mathbf{h}_{e}
\end{array}\right]+\left[\begin{array}{c}
\varphi\left(\mathbf{h}_{e}+\overline{\mathbf{h}}\right) \\
\mathbf{I}
\end{array}\right] u(t) \\
& =\widetilde{\mathcal{Z}}\left(\widetilde{\mathbf{z}}_{e}\right)+\Phi\left(\mathbf{h}_{e}\right) u(t) .
\end{aligned}
$$


Note that since $u(t) \neq 0$, the CSI $\mathbf{h}(t)$ is time varying even after the transient and its impact on the stability of the virtual system is captured by the virtual exogenous input $\Phi\left(\mathbf{h}_{e}\right) u(t)$ in [19]. The following theorem summarizes the stability results for $\widetilde{\mathcal{Z}}_{e}(u)$.

Theorem 2 (Stability of $\widetilde{\mathcal{Z}}_{e}(u)$ for Time Varying CSI): Given $\left\|\Phi\left(\mathbf{h}_{e}\right)\right\| \leq \gamma, \overline{\|u(t)\|^{2}} \leq \alpha^{2}$, the average trajectory $\overline{\left\|\widetilde{\mathbf{z}}_{e}\right\|^{2}}$ of the virtual dynamic system $\widetilde{\mathcal{Z}}_{e}(u)$ in $(19)$ satisfies

$$
\overline{\left\|\widetilde{\mathbf{z}}_{e}\right\|^{2}}=\lim _{T \rightarrow \infty} \frac{1}{T} \int_{0}^{T}\left\|\widetilde{\mathbf{z}}_{e}(t)\right\|^{2} d t \leq \frac{a_{4}^{2} \gamma^{2}}{a_{3}^{2}} \alpha^{2}
$$

where $\overline{\|u(t)\|^{2}}=\lim _{T \rightarrow \infty} \frac{1}{T} \int_{0}^{T}\|u(t)\|^{2} d t$.

Proof: Please refer to Appendix $\mathrm{D}$ for the proof.

Note that $\overline{\|u(t)\|^{2}}$ denotes the average exogenous excitation of $u(t)$ to the virtual system $\widetilde{\mathcal{Z}}_{e}$ and $\overline{\left\|\widetilde{\mathbf{z}}_{e}\right\|^{2}}$ denotes the average tracking error of the system. Based on the result in Theorem 2. the following corollary summarizes the convergence performance of the primal-dual algorithm for a strongly concave-convex saddle point problem in time varying CSI.

Corollary 1: (Convergence Performance of Primal-Dual Algorithms for Strongly Concave-Convex Saddle Point Problems): Suppose $\|\varphi(\mathbf{h})\| \leq \gamma_{0}$ for all $\mathbf{h} \in \mathcal{H}$ and $\overline{\|u(t)\|^{2}} \leq \alpha^{2}$, the average tracking error $\overline{\left\|\widetilde{\mathbf{x}}_{e}\right\|^{2}}$ of the primal-dual algorithm under time varying CSI satisfies $\overline{\left\|\widetilde{\mathbf{x}}_{e}\right\|^{2}}=$ $\lim _{T \rightarrow \infty} \frac{1}{T} \int_{0}^{T}\left\|\widetilde{\mathbf{x}}_{e}(t)\right\|^{2} d t \leq \frac{a_{4}^{2}\left(\gamma_{0}^{2}+1\right)}{a_{3}^{2}} \alpha^{2}$.

Proof: Since $\left\|\Phi\left(\mathbf{h}_{e}\right)\right\|=\sqrt{\left\|\varphi\left(\mathbf{h}_{e}+\overline{\mathbf{h}}\right)\right\|^{2}+1} \leq \sqrt{\gamma_{0}^{2}+1}$, by Theorem 2, the average trajectory of the corresponding virtual dynamic system $\widetilde{\mathcal{Z}}_{e}(u)$ for the primal-dual iterative algorithm converges to $\overline{\left\|\widetilde{\mathbf{z}}_{e}\right\|^{2}}=\lim _{T \rightarrow \infty} \frac{1}{T} \int_{0}^{T}\left\|\widetilde{\mathbf{z}}_{e}(t)\right\|^{2} d t \leq \frac{a_{4}^{2}\left(\gamma_{0}^{2}+1\right)}{a_{3}^{2}} \alpha^{2}$. As $\left\|\widetilde{\mathbf{x}}_{e}\right\| \leq\left\|\widetilde{\mathbf{z}}_{e}\right\|$ the average trajectory $\overline{\left\|\widetilde{\mathbf{x}}_{e}(t)\right\|^{2}}$ of the error process $\widetilde{\mathcal{X}}_{e}$ converges to $\frac{a_{4}^{2}\left(\gamma_{0}^{2}+1\right)}{a_{3}^{2}} \alpha^{2}$, and equivalently, the average tracking error $\overline{\left\|\widetilde{\mathbf{x}}_{e}\right\|^{2}}$ of the primal-dual algorithm satisfies $\lim _{T \rightarrow \infty} \frac{1}{T} \int_{0}^{T}\left\|\widetilde{\mathbf{x}}_{e}(t)\right\|^{2} d t \leq \frac{a_{4}^{2}\left(\gamma_{0}^{2}+1\right)}{a_{3}^{2}} \alpha^{2}$. This completes the proof of Corollary 1 .

Note that the average tracking error $\overline{\left\|\widetilde{\mathbf{x}}_{e}\right\|^{2}}$ depends on $\alpha^{2}, \gamma_{0}$ and $a_{4} / a_{3}$ which represents how fast the CSI changes, how sensitive the equilibrium $\widetilde{\mathbf{x}}^{*}(t)$ is w.r.t. the change of CSI $\mathbf{h}(t)$, and how fast the algorithm converges, respectively. For instance, the average tracking error $\overline{\left\|\widetilde{\mathbf{x}}_{e}\right\|^{2}}$ will be smaller if the CSI is slowly changing (i.e. $\overline{\|u(t)\|^{2}}$ is small) or the convergence rate of the underlying primal-dual algorithm (under time invariant CSI) is fast (i.e. smaller $a_{4} / a_{3}$ ).

\section{B. Numerical Example}

We shall illustrate the application of Theorem 2 using a numerical example from Section II-B1. Consider a 2-antenna system; the fading channels are given by $\mathbf{H}_{1}, \mathbf{H}_{2} \in \mathbb{C}^{2 \times 2}$, and $\mathbf{h}=\operatorname{vec}\left(\left[\mathbf{H}_{1} \mathbf{H}_{2}\right]\right) \in$ $\mathbb{C}^{8}$. We choose the channel model in (9) as $A=-a I, \overline{\mathbf{h}}=\mathbf{1}$ and $u(t)=\sqrt{2 a} w(t)$, where $w(t)$ is a zero-mean unit-variance white Gaussian process. This corresponds to a standard AR Gaussian fading process. The parameter $a$ controls the time-correlation of the CSI and the CSI $\mathbf{h}(t)$ has unity 
variance. The primal-dual algorithm for the specific example is given by

$$
\left[\begin{array}{c}
\frac{d \operatorname{vec}(\mathbf{Q})}{d t} \\
\frac{d \operatorname{vec}(\mathbf{Z})}{d t}
\end{array}\right]=\left[\begin{array}{c}
\left\{\left(\frac{\partial}{\partial \operatorname{vec}^{T}(\mathbf{Q})} C(\mathbf{Q}, \mathbf{Z} ; \mathbf{h}(t))\right)^{T}\right\}_{\mathcal{Q}} \\
\left\{\left(-\frac{\partial}{\partial \operatorname{vec}^{T}(\mathbf{Z})} C(\mathbf{Q}, \mathbf{Z} ; \mathbf{h}(t))\right)^{T}\right\}_{\mathcal{J}}
\end{array}\right] \triangleq \widetilde{f}(\widetilde{\mathbf{x}} ; \mathbf{h}(t))
$$

where $\mathcal{Q}=\left\{\mathbf{Q} \in \mathbb{C}^{2 \times 2}: \mathbf{Q} \succeq 0, \operatorname{tr}(\mathbf{Q}) \leq P_{T}\right\}$ and $\mathcal{J}=\left\{\mathbf{Z} \in \mathbb{C}^{2 \times 2}: \mathbf{Z} \succeq 0, \operatorname{tr}(\mathbf{Z}) \leq P_{J}\right\}$ are the feasible sets for the transmission covariance matrices, projections $\{\cdot\}_{\mathcal{Q}}$ and $\{\cdot\}_{\mathcal{J}}$ are to restrict the searching directions within the feasible sets $\mathcal{Q}$ and $\mathcal{J}$, respectively, and $\widetilde{\mathbf{x}}=\left[\operatorname{vec}^{T}(\mathbf{Q}) \operatorname{vec}^{T}(\mathbf{Z})\right]^{T}$. In this numerical example, the power constraints are set to be $P_{T}=P_{J}=10$.

Correspondingly, the error dynamic system $\widetilde{\mathcal{X}}_{e}$ and the virtual dynamic system $\widetilde{\mathcal{Z}}_{e}(u)$ is given by (12) and (19) respectively, with $\widetilde{f}(\widetilde{\mathbf{x}} ; \mathbf{h}(t))$ defined in (21). The equilibrium point is defined as $\widetilde{f}\left(\widetilde{\mathbf{x}}^{*} ; \mathbf{h}(t)\right)=\mathbf{0}$ and the function $\varphi(\mathbf{h}) \triangleq \frac{\partial}{\partial \mathbf{h}} \widetilde{\mathbf{x}}^{*}(\mathbf{h})=\left(\frac{\partial}{\partial \widetilde{\mathbf{x}}} \widetilde{f}\left(\widetilde{\mathbf{x}}^{*} ; \mathbf{h}\right)\right)^{-1} \frac{\partial}{\partial \mathbf{h}} \widetilde{f}\left(\widetilde{\mathbf{x}}^{*} ; \mathbf{h}\right)$.

It can be numerically verified that $\|\varphi(\mathbf{h})\| \leq 11.22$ for all possible $\mathbf{h} \in \mathcal{H} \subseteq \mathbb{C}^{8}$, and the condition (15) in Lemma 2 is satisfied by choosing $\kappa=1 \mathrm{sec}^{-1}$. Hence by Theorem 2, we conclude that the average tracking error $\frac{1}{T} \int_{0}^{T}\left\|\widetilde{\mathbf{x}}(t)-\widetilde{\mathbf{x}}^{*}(t)\right\|^{2} d t$ converges to $a_{4}^{2} \gamma^{2} \alpha^{2} / a_{3}^{2}$ with $\gamma=\sqrt{\|\varphi(\mathbf{h})\|^{2}+1} \leq$ 11.26, $\alpha^{2}=16 a$ and $a_{4} / a_{3}=0.222$ (when the CSI parameter $a=0.02 \mathrm{~ms}^{-1}$ ).

\section{Convergence Analysis of Degraded Saddle Point Problems under Time VARYING CSI}

In Section III. we have focused on the case where the objective function $\mathcal{L}(\mathbf{x}, \lambda)$ is strongly concave in $\mathrm{x}$ and strongly convex in $\lambda$. This property provides exponential convergence rate to the saddle point $\left(\mathbf{x}^{*}, \lambda^{*}\right)$ of (1). In this section we shall extend the results to deal with the case when one of the Hessians is only semidefinite. Without loss of generality, we assume $\nabla_{x}^{2} \mathcal{L} \preceq-M_{x} \mathbf{I}$ for $M_{x}>0$ and $\nabla_{\lambda}^{2} \mathcal{L} \succeq 0$, and this corresponds to the case where the objective function $\mathcal{L}(\mathbf{x}, \lambda)$ is strongly concave in $\mathbf{x}$ and only convex in $\lambda$. We call such a saddle point problem the degraded saddle point problem and the associated virtual dynamic systems the degraded virtual dynamic systems. Examples of degraded saddle point problems include the primal-dual iterations (single time scale) of convex optimization problems. For instance, the Lagrangian function (7) of the NUM problem (6) is strongly concave in the primal variables $\mathbf{r}$ and $\mathbf{p}$ but only convex in the LM $\lambda$.

\section{A. Stability Analysis of the Degraded Virtual Dynamic System}

Similarly, we first consider the case when the CSI is time invariant $(\dot{\mathbf{h}}=0)$. For degraded saddle point problems, the exponential stability property of the associated virtual dynamic system $\widetilde{\mathcal{X}}_{e}$ in Lemma 1 does not hold. We have a partial exponential stability property summarized in the following. 
Lemma 3 (Partial Exponential Stability of $\widetilde{\mathcal{X}}_{e}$ with Time Invariant CSI): When the CSI is time invariant, the equilibrium point $\widetilde{\mathbf{x}}_{e}^{*}=\left(\mathbf{x}_{e}^{*}, \lambda_{e}^{*}\right)=0$ of the virtual dynamic system $\widetilde{\mathcal{X}}_{e}$ in 12 is partially exponentially stable w.r.t. $\mathbf{x}_{e}^{*}$ for all $\mathbf{h} \in \mathcal{H}$, i.e., there exists a Lyapunov function $V_{x}: \mathbb{R}^{n+m} \times \mathbb{R}^{q} \mapsto \mathbb{R}$ defined on the virtual state $\widetilde{\mathbf{x}}_{e}$ that satisfies

$$
\begin{gathered}
c_{1}\left\|\widetilde{\mathbf{x}}_{e}\right\|^{2} \leq V_{x}\left(\widetilde{\mathbf{x}}_{e}\right) \leq c_{2}\left\|\widetilde{\mathbf{x}}_{e}\right\|^{2} \\
\dot{V}_{x}\left(\widetilde{\mathbf{x}}_{e}\right)=\frac{\partial V}{\partial \widetilde{\mathbf{x}}_{\mathbf{e}}} \widetilde{f}_{e}\left(\widetilde{\mathbf{x}}_{e} ; \mathbf{h}\right) \leq-c_{3}\left\|\mathbf{x}_{e}\right\|^{2} \\
\left\|\frac{\partial V_{x}}{\partial \widetilde{\mathbf{x}}_{e}}\right\| \leq c_{4}\left\|\widetilde{\mathbf{x}}_{e}\right\| .
\end{gathered}
$$

The proof is similar to that in Appendix $\mathrm{B}$, by simply taking a Lyapunov function $V_{x}\left(\widetilde{\mathbf{x}}_{e}\right)=$ $\frac{1}{2 \kappa} \widetilde{\mathbf{x}}_{e}^{T} \widetilde{\mathbf{x}}_{e}$, and letting $M_{\lambda}=0$. For this Lyapunov function, $c_{1}=c_{2}=\frac{1}{2 \kappa}, c_{3}=2 M_{x}$ and $c_{4}=1 / \kappa$. Note that, instead of possessing exponential stability on the joint state $\widetilde{\mathbf{x}}_{e}^{*}=\left(\mathbf{x}_{e}^{*}, \lambda_{e}^{*}\right)$, the degraded virtual dynamic system just has exponential stability on $\mathbf{x}_{e}^{*}$ as shown in 23).

Similarly, we study the stability of the virtual dynamic system $\dot{\widetilde{\mathbf{x}}}_{e}=\widetilde{f}_{e}\left(\widetilde{\mathbf{x}}_{e} ; \mathbf{h}(t)\right)-\varphi(\mathbf{h}) \dot{\mathbf{h}}(t)$ with a quasi-time varying CSI $\mathbf{h}(t)$ for $u(t)=0$. Since we have exponential stability only on $\mathbf{x}_{e}^{*}$ but not on $\lambda_{e}^{*}$, we define a partial state as $\mathbf{z}_{e}=\left(\mathbf{x}_{e}, \mathbf{h}_{e}\right)$. As a result, the trajectory of the partial state $\mathbf{z}_{e}$ satisfies the following virtual dynamic system $\mathcal{Z}_{e}$ :

$$
\mathcal{Z}_{e}: \quad \dot{\mathbf{z}}_{e}=\mathcal{Z}\left(\mathbf{z}_{e}\right):=\left[\begin{array}{c}
f_{e}\left(\mathbf{x}_{e}, \lambda_{e}=0 ; \mathbf{h}_{e}+\overline{\mathbf{h}}\right)+\varphi_{x}\left(\mathbf{h}_{e}+\overline{\mathbf{h}}\right) A \mathbf{h}_{e} \\
A \mathbf{h}_{e}
\end{array}\right]
$$

where $f_{e}\left(\mathbf{x}_{e}, \lambda_{e} ; \mathbf{h}(t)\right)=\left(\frac{\partial}{\partial \mathbf{x}_{\mathrm{e}}} \mathcal{L}\left(\mathbf{x}_{e}+\mathbf{x}^{*}, \lambda_{e}+\lambda^{*} ; \mathbf{h}(t)\right)\right)^{T}$ and $\varphi_{x}(\mathbf{h})=\frac{\partial}{\partial \mathbf{h}} \mathbf{x}^{*}(\mathbf{h})=\frac{\partial}{\partial \mathbf{h}} \psi_{x}(\mathbf{h})$. The following lemma summarizes the stability results for the virtual system $\dot{\mathbf{z}}_{e}=\mathcal{Z}\left(\mathbf{z}_{e}\right)$.

Lemma 4 (Partial Exponential Stability of $\mathcal{Z}_{e}$ under Quasi-Time Varying CSI): Suppose the following inequality holds for all $\mathbf{h} \in \mathcal{H}$ :

$$
\left\|\varphi_{x}(\mathbf{h}) A\right\|<\kappa \min \left\{2 M_{x},-\lambda_{\max }(A)\right\}
$$

where $\lambda_{\max }(A)$ denotes the largest eigenvalue of $A$. Then the system $\mathcal{Z}_{e}$ in 25 is partially exponentially stable, i.e., there exists a Lyapunov function for the joint state $\mathbf{z}_{e}$ satisfying

$$
\begin{gathered}
a_{1}\left\|\mathbf{z}_{e}\right\|^{2} \leq V\left(\mathbf{z}_{\mathbf{e}}\right) \leq a_{2}\left\|\mathbf{z}_{e}\right\|^{2} \\
\dot{V}\left(\mathbf{z}_{e}\right)=\frac{\partial V}{\partial \mathbf{z}_{e}} Z\left(\mathbf{z}_{e}\right) \leq-a_{3}\left\|\mathbf{z}_{e}\right\|^{2} \\
\left\|\frac{\partial V}{\partial \mathbf{z}_{e}}\right\| \leq a_{4}\left\|\mathbf{z}_{e}\right\| .
\end{gathered}
$$

Proof: Please refer to Appendix E for the proof. 
Lemma 4 suggests that as long as the transient of quasi-time varying $\mathbf{h}(t)$ is not changing too fast (i.e. $A$ has small eigenvalues) and the sensitivity of the primal part of the equilibrium $\mathrm{x}_{e}^{*}$ w.r.t. the change of $\mathbf{h}(t)$ is small (i.e. small $\left\|\varphi_{x}(\mathbf{h})\right\|$ ), the virtual dynamic system $\mathcal{Z}_{e}$ still possesses globally exponential stability on the partial state $\mathbf{z}_{e}=\left(\mathbf{x}_{e}, \mathbf{h}_{e}\right)$.

Finally, we consider the stability result of the degraded virtual dynamic system under the time varying CSI model in (9). The degraded virtual dynamic system $\mathcal{Z}_{e}(u)$ is given by

$$
\begin{aligned}
\mathcal{Z}_{e}(u): \quad \dot{\mathbf{z}}_{e} & =\left[\begin{array}{c}
f_{e}\left(\mathbf{x}_{e}, \lambda_{e}=0 ; \mathbf{h}\right)+\varphi_{x}\left(\mathbf{h}_{e}+\overline{\mathbf{h}}\right) A \mathbf{h}_{e} \\
A \mathbf{h}_{e}
\end{array}\right]+\left[\begin{array}{c}
\varphi_{x}\left(\mathbf{h}_{e}+\overline{\mathbf{h}}\right) \\
\mathbf{I}
\end{array}\right] u(t) \\
& =\mathcal{Z}\left(\mathbf{z}_{e}\right)+\Phi_{x}\left(\mathbf{h}_{e}\right) u(t) .
\end{aligned}
$$

The stability result is summarized in the following theorem.

Theorem 3 (Stability of $\mathcal{Z}_{e}(u)$ for Time Varying CSI): Given $\left\|\Phi_{x}\left(\mathbf{h}_{e}\right)\right\| \leq \gamma_{x}$, and $\overline{\|u(t)\|^{2}} \leq \alpha^{2}$, the average trajectory $\overline{\left\|\mathbf{z}_{e}\right\|^{2}}$ of the degraded virtual dynamic system $\mathcal{Z}_{e}(u)$ satisfies

$$
\overline{\left\|\mathbf{z}_{e}\right\|^{2}}=\frac{1}{T} \int_{0}^{T}\left\|\mathbf{z}_{e}(t)\right\|^{2} d t \leq \frac{a_{4}^{2} \gamma_{x}^{2}}{a_{3}^{2}} \alpha^{2}
$$

where $\overline{\|u(t)\|^{2}}=\lim _{T \rightarrow \infty} \frac{1}{T} \int_{0}^{T}\|u(t)\|^{2} d t$.

The proof can be derived similarly from that of Theorem 2 using the property of Lemma 4. As a result, we summarize the convergence performance of the primal-dual iterative algorithm for a degraded saddle point problem in the following corollary.

Corollary 2: (Convergence Performance of Primal-Dual Algorithms for Degraded Saddle Point Problems): Suppose $\varphi_{x}(\mathbf{h}) \leq \bar{\gamma}_{x}$ for all $\mathbf{h} \in \Gamma$ and $\overline{\|u(t)\|^{2}} \leq \alpha^{2}$, the average tracking error $\overline{\left\|\mathbf{x}_{e}\right\|^{2}}$ for the primal-dual algorithm under time varying CSI satisfies $\overline{\left\|\mathbf{x}_{e}\right\|^{2}}=\lim _{T \rightarrow \infty} \frac{1}{T} \int_{0}^{T}\left\|\mathbf{x}_{e}(t)\right\|^{2} d t \leq$ $\frac{a_{4}^{2}\left(\bar{\gamma}_{x}^{2}+1\right)}{a_{3}^{2}} \alpha^{2}$.

The proof can be derived similarly from that of Corollary 1 , by applying Theorem 3 and using $\left\|\Phi_{x}\left(\mathbf{h}_{e}\right)\right\|=\sqrt{\left\|\varphi_{x}\left(\mathbf{h}_{e}+\overline{\mathbf{h}}\right)\right\|^{2}+1} \leq \sqrt{\bar{\gamma}_{x}^{2}+1}$ and $\left\|\mathbf{x}_{e}\right\| \leq\left\|\mathbf{z}_{e}\right\|$.

Together with Lemma 4, the above theorem and corollary establish sufficient conditions on the convergence performance of the a primal-dual algorithm for a degraded saddle point problem under time varying CSI. It shows that once the corresponding virtual dynamic system $\dot{\mathbf{z}}_{e}=\mathcal{Z}_{u}\left(\mathbf{z}_{e}, u(t)\right)$ is exponentially stable at $\mathbf{z}_{e}^{*}=0$ for $u(t)=0$, the system is stable for general $u(t)$ as long as $\overline{\|u(t)\|^{2}}$ is bounded. Correspondingly, the tracking error of the primal-dual algorithm under time varying CSI is bounded and scaled according to $\mathcal{O}\left(\overline{\|u(t)\|^{2}}\right)$.

Note that, due to the degraded saddle point problem in the dual variable $\lambda$, the average error bound $\frac{a_{4}^{2}\left(\bar{\gamma}_{x}^{2}+1\right)}{a_{3}^{2}} \alpha^{2}$ in Corollary 2 does not include the dual variable $\lambda_{e}$, and hence we cannot bound the tracking error of the dual variables. This is because due to the semidefinite Hessian matrix $\nabla_{\lambda} \mathcal{L} \succeq 0$, the dual variable $\lambda$ in the primal-dual iterative algorithm (2)-(3) may not converge exponentially fast 
to the saddle point $\lambda^{*}$. However, this result is still meaningful because, in many applications such as constrained optimizations, we are mainly concerned about the behavior of the primal variable $\mathrm{x}$. We shall illustrate with an example in the next section.

\section{B. Numerical Example}

We illustrate an application of Theorem 3 using a numerical example in Section II-B2. Consider a simple wireless network with 3 nodes depicted in Fig. 2, where the collection of traffic flows is given by $\mathcal{C}=\{(1,2),(1,3),(2,3)\}$, and the sets of links are $L(1,2)=\{1\}, L(1,3)=\{1,2\}$ and $L(2,3)=\{2\} . P_{t 1}$ and $P_{t 2}$ denote the total power allocated to link 1 and link 2, respectively, and $h_{1}$ and $h_{2}$ are the corresponding channel gains. Let $\mathbf{h}=\left[h_{1} h_{2}\right]^{T}$. Our CSI dynamic is modeled in (9), with $A=-a I$ and $u(t)=\sqrt{2 a} w(t)$ where $w(t)$ is a zero-mean unit-variance white Gaussian process. The variance of the CSI $\mathbf{h}(t)$ is normalized to unity and the parameter $a$ controls the time-correlation of the CSI. The average receiving SNR is normalized to $10 \mathrm{~dB}$. We consider $U_{s d}\left(r_{s d}\right)=\log \left(1+r_{s d}\right)$ as the cost function, and the link capacity function is given by $c_{l}\left(P_{t l}, h_{l}\right)=\log \left(1+h_{l}^{2} P_{t l}\right), \forall l=1,2$. The Lagrangian saddle point problem for this specific example is given by

$\min _{\lambda \succeq 0} \max _{\mathbf{r}} \mathcal{L}(\mathbf{r}, \lambda)=\sum_{(s, d) \in \mathcal{C}} \log \left(1+r_{s d}\right)-\sum_{l=1,2} \lambda_{l}\left(\sum_{(s, d): l \in L(s, d)} r_{s d}-\log \left(1+h_{l}^{2} P_{t l}\right)\right)+\sum_{(s, d) \in \mathcal{C}} \lambda_{r}^{(s d)} r_{s d}$.

The error dynamic system and the degraded virtual dynamic system are given by (12) and 25, respectively. Specifically, let $\mathbf{r}=\left[\begin{array}{lll}r_{12} & r_{13} & r_{23}\end{array}\right]^{T}$ be the primal variable and $\boldsymbol{\lambda}$ be the collection of the dual variables. Then $\widetilde{\mathbf{x}}_{e}=\widetilde{\mathbf{x}}-\widetilde{\mathbf{x}}^{*}, \widetilde{\mathbf{x}}=(\mathbf{r}, \lambda), \mathbf{z}_{e}=(\mathbf{r}, \mathbf{h}), \Phi_{\mathbf{r}}(\mathbf{h})=\left[\varphi_{\mathbf{r}}(\mathbf{h}) ; I\right]$, and $\varphi(\mathbf{h})=\frac{\partial}{\partial \mathbf{h}} \widetilde{\mathbf{x}}^{*}(\mathbf{h})=\left(\frac{\partial F}{\partial \widetilde{\mathbf{x}}}\right)^{-1} \frac{\partial F}{\partial \mathbf{h}}$ which is derived by the implicit function theorem, where the implicit function $F\left(\mathbf{r}^{*}, \lambda^{*}\right)=0$ is given by the KKT conditions

$$
F\left(\mathbf{r}^{*}, \lambda^{*}\right)=\left[\begin{array}{c}
\nabla_{\mathbf{r}} \mathcal{L}\left(\mathbf{r}^{*}, \lambda^{*}\right) \\
\lambda_{l}^{*}\left(\sum r_{s d}^{*}-c_{l}\left(P_{t l}, h_{l}\right)\right) \\
\lambda_{r}^{(s d) *} r_{s d}^{*}
\end{array}\right]=\mathbf{0} .
$$

Numerical results suggest that $\left\|\varphi_{\mathbf{r}}(\mathbf{h})\right\| \leq 2.2$, and hence the constants in Lemma 3 can be chosen as $\kappa=0.5 \mathrm{sec}^{-1}$, such that condition 26 in Lemma 4 is satisfied. Thus we can apply Corollary 2, which shows that the average tracking error for the rate allocation variables $\overline{\left\|\mathbf{r}_{e}\right\|^{2}}=$ $\lim _{T \rightarrow \infty} \frac{1}{T} \int_{0}^{T}\left\|\mathbf{r}_{e}(t)\right\|^{2} d t \leq \frac{a_{4}^{2}}{a_{3}^{2}} \gamma_{r}^{2} \alpha^{2}$, where $\gamma_{r} \leq \sqrt{\left\|\varphi_{r}(\mathbf{h})\right\|^{2}+1} \leq 2.42, \alpha^{2}=4 a$, and $a_{4} / a_{3}=$ 1.69 (when the CSI parameter $a=0.04 \mathrm{~ms}^{-1}$ ).

\section{Adaptive Compensation for the Primal Dual Algorithms in Time Varying CHANNELS}

In the previous sections, we have analyzed the convergence behavior of the primal-dual algorithms under time varying CSI. In this section, we consider modifying the primal-dual iterations to reduce 
the tracking error in time varying CSI. To this end, we shall introduce a compensation term in the iteration to offset the exogenous excitation to the virtual dynamic system in (19) and (30). In the following, we shall first discuss the construction of the compensation term. Based on that, we shall obtain a low complexity distributive implementation for the compensation method.

\section{A. Adaptive Compensation for the Primal Dual Algorithm}

We have shown in Theorem 2 and Theorem 3 that the average tracking error of a primal-dual algorithm under time varying CSI is $\mathcal{O}\left(\alpha^{2} \gamma^{2}\right)$ where $\alpha^{2} \gamma^{2}$ is the square average of the norm of the exogenous input $\Phi\left(\mathbf{h}_{e}\right) u(t)$ to the virtual dynamic system $\widetilde{\mathcal{Z}}_{e}$. Consider a particle trapped in an energy well given by the Lyapunov function $5\left(\widetilde{\mathbf{z}}_{e}\right)$. The presence of the exogenous input term $\Phi\left(\mathbf{h}_{e}\right) u(t)$ to the system $\widetilde{\mathcal{Z}}_{e}$ excites the particle and moves it away from the equilibrium position, and how far the particle can be moved depends on the exogenous excitation energy induced by $u(t)$. This phenomenon is illustrated in Fig. 4. As a result, one way to reduce the tracking error is to compensate the disturbance from the exogenous input $\Phi\left(\mathbf{h}_{e}\right) u(t)$. We thus introduce a compensation term $\widehat{\Phi}\left(\widetilde{\mathbf{z}}_{e}\right) u(t)$ to the virtual dynamic system $\widetilde{\mathcal{Z}}_{e}(u)$ in 19 as follows,

$$
\widetilde{\mathcal{Z}}_{e}(\widehat{u}): \quad \dot{\tilde{\mathbf{z}}}_{e}=\widetilde{\mathcal{Z}}\left(\widetilde{\mathbf{z}}_{e}\right)+\Phi\left(\mathbf{h}_{e}\right) u(t)-\widehat{\Phi}\left(\widetilde{\mathbf{z}}_{e}\right) u(t)
$$

where $\widehat{\Phi}\left(\widetilde{\mathbf{z}}_{e}\right)=\left[\begin{array}{c}\widehat{\varphi}\left(\widetilde{\mathbf{x}}_{e}, \mathbf{h}_{e}\right) \\ \mathbf{I}\end{array}\right]$ is the compensation term to be derived. Obviously, if we could set $\widehat{\Phi}\left(\widetilde{\mathbf{z}}_{e}\right)=\Phi\left(\mathbf{h}_{e}\right)$, the impact of the exogenous input $\Phi\left(\mathbf{h}_{e}\right) u(t)$ can be totally suppressed and the virtual dynamic system $\widetilde{\mathcal{Z}}_{e}(\widehat{u})$ will be free from exogenous excitation. However, since we do not have closed form expression for the saddle point $\widetilde{\mathbf{x}}^{*}(\mathbf{h}), \Phi\left(\mathbf{h}_{e}\right)$ cannot be obtained during the iteration.

On the other hand, suppose that there exists a function $F: \mathbb{R}^{n} \times \mathbb{R}_{+}^{m} \mapsto \mathbb{R}^{n+m} \in \mathcal{C}^{1}$ such that $F\left(\widetilde{\mathbf{x}}^{*} ; \mathbf{h}\right)=0$ and $\frac{\partial}{\partial \widetilde{\mathbf{x}}} F\left(\widetilde{\mathbf{x}}^{*} ; \mathbf{h}\right)$ is non-singular for all $\widetilde{\mathbf{x}}^{*}(\mathbf{h})$ and $\mathbf{h} \in \mathcal{H} \subset \mathbb{R}^{q}$. Using the implicit function theorem, the function $\Phi\left(\mathbf{h}_{e}\right)$ in 31 is given by

$$
\Phi\left(\mathbf{h}_{e}\right)=\left[\begin{array}{c}
-\left[\frac{\partial}{\partial \widetilde{\mathbf{x}}} F\left(\widetilde{\mathbf{x}}^{*}\left(\mathbf{h}_{e}+\overline{\mathbf{h}}\right) ; \mathbf{h}_{e}+\overline{\mathbf{h}}\right)\right]^{-1} \frac{\partial}{\partial \mathbf{h}} F\left(\widetilde{\mathbf{x}}^{*}\left(\mathbf{h}_{e}+\overline{\mathbf{h}}\right) ; \mathbf{h}_{e}+\overline{\mathbf{h}}\right) \\
\mathbf{I}
\end{array}\right]
$$

Note that the function $F($.$) can be found by the optimality conditions of the saddle point problem.$ For example, it can be chosen as $\widetilde{f}\left(\widetilde{\mathbf{x}}^{*} ; \mathbf{h}(t)\right)=0$ by the definition of equilibrium point (Definition 2), where $\tilde{f}(\cdot)$ is given in (10). Using (32), we can estimate $\Phi\left(\mathbf{h}_{e}\right)$ by

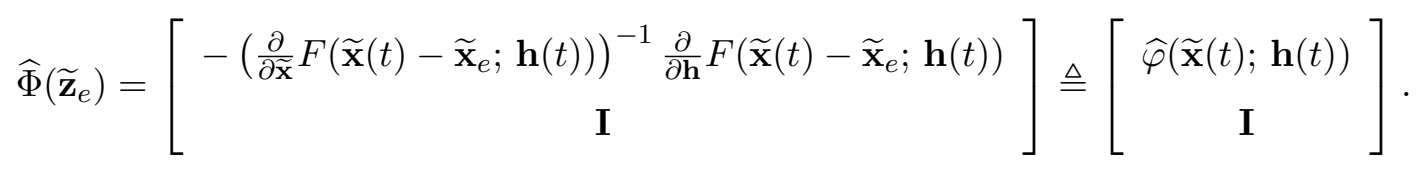

\footnotetext{
${ }^{5}$ The Lyapunov function can be interpreted as the energy function of the system state $\widetilde{\mathbf{z}}_{e}$.
} 
Note that the joint state $\widetilde{\mathbf{z}}_{e}=\left(\widetilde{\mathbf{x}}_{e}, \mathbf{h}_{e}\right)$ and $\widetilde{\mathbf{x}}(t)$ are the algorithm outputs at the $t$-th algorithm iteration in 34)-35. The estimate $\widehat{\Phi}\left(\widetilde{\mathbf{z}}_{e}\right)$ in 33 is quite accurate when the algorithm trajectory $\widetilde{\mathbf{x}}(t)$ is close enough to the equilibrium $\widetilde{\mathbf{x}}^{*}(\mathbf{h}(t))$ (i.e. $\left\|\widetilde{\mathbf{x}}_{e}\right\|$ is small enough). As a result, the corresponding primal-dual algorithm iterations with compensation is given by

$$
\begin{aligned}
& \dot{\mathbf{x}}=\frac{d \mathbf{x}}{d t}=\left[\frac{\partial}{\partial \mathbf{x}} \mathcal{L}(\mathbf{x}, \lambda ; \mathbf{h}(t))+\widehat{\varphi}_{\mathbf{x}}(\mathbf{x}, \lambda ; \mathbf{h}(t))^{T} \dot{\mathbf{h}}(t)\right]^{T} \\
& \dot{\lambda}=\frac{d \lambda}{d t}=\left[\left(-\frac{\partial}{\partial \lambda} \mathcal{L}(\mathbf{x}, \lambda ; \mathbf{h}(t))+\widehat{\varphi}_{\lambda}(\mathbf{x}, \lambda ; \mathbf{h}(t))^{T} \dot{\mathbf{h}}(t)\right)^{T}\right]_{\lambda}^{+}
\end{aligned}
$$

where $\widehat{\varphi}_{\mathbf{x}}(\mathbf{x}, \lambda ; \mathbf{h}(t))$ and $\widehat{\varphi}_{\lambda}(\mathbf{x}, \lambda ; \mathbf{h}(t))$ are the primal and dual parts of the compensation term $\widehat{\varphi}(\widetilde{\mathbf{x}} ; \mathbf{h}(t))$.

The compensation terms in (34)-35 can also be interpreted as a predictor on where the saddle point $\widetilde{\mathbf{x}}^{*}(\mathbf{h})$ moves as illustrated in Fig. 5. We summarize the performance of the proposed algorithm in the following theorem.

Theorem 4: (Tracking Performance of the Compensated Primal-Dual Iteration for Strongly ConcaveConvex Saddle Point Problems): Suppose that the compensation term $\widehat{\varphi}(\widetilde{\mathbf{x}} ; \mathbf{h}(t))$ is Lipschitz continuous on $\widetilde{\mathbf{x}}$ satisfying $\left\|\widehat{\varphi}(\widetilde{\mathbf{x}}(t) ; \mathbf{h}(t))-\widehat{\varphi}\left(\widetilde{\mathbf{x}}^{*}(t) ; \mathbf{h}(t)\right)\right\| \leq L\left\|\widetilde{\mathbf{x}}_{e}(t)\right\|$ for all $\mathbf{h}(t) \in \mathcal{H} \subseteq \mathbb{R}^{q}$ and $\lambda(t) \in \mathbb{R}_{+}^{m}$, and $\overline{\|u(t)\|}=\lim _{T \rightarrow \infty} \frac{1}{T} \int_{0}^{T}\|u(t)\| d t=\beta<\frac{a_{3}}{a_{4} L}$. Then the average tracking error $\overline{\left\|\widetilde{\mathbf{x}}_{e}\right\|^{2}}$ of the proposed algorithm (34)-35) converges to 0 .

Proof: Please refer to Appendix $\mathrm{F}$ for the proof.

Similarly, for a degraded saddle point problem, the same proposed algorithm (34)- 35 can be applied, and the degraded virtual dynamic system $\mathcal{Z}_{e}(\widehat{u})$ is given by

$$
\mathcal{Z}_{e}(\widehat{u}): \quad \dot{\mathbf{z}}_{e}=\mathcal{Z}_{e}\left(\mathbf{z}_{e}\right)+\left(\Phi_{x}\left(\mathbf{h}_{e}\right)-\widehat{\Phi}_{x}\left(\widetilde{\mathbf{z}}_{e}\right)\right) u(t)
$$

where

$$
\widehat{\Phi}_{x}\left(\widetilde{\mathbf{z}}_{e}\right)=\left[\begin{array}{c}
\widehat{\varphi}_{\mathbf{x}}(\mathbf{x}, \lambda ; \mathbf{h}(t)) \\
\mathbf{I}
\end{array}\right]
$$

and the associated performance is summarized below.

Corollary 3: (Tracking Performance of the Compensated Primal-Dual Iteration for Degraded Saddle Point Problems): Suppose the compensation term $\widehat{\varphi}_{\mathbf{x}}(\mathbf{x}, \lambda ; \mathbf{h}(t))$ is Lipschitz continuous on $\mathbf{x}$ satisfying $\| \widehat{\varphi}_{\mathbf{x}}\left(\mathbf{x}(t), \lambda(t) ; \mathbf{h}(t)-\widehat{\varphi}_{\mathbf{x}}\left(\mathbf{x}^{*}(t), \lambda(t) ; \mathbf{h}(t)\right)\left\|\leq L_{x}\right\| \mathbf{x}_{e}(t) \|\right.$ for all $\mathbf{h}(t) \in \mathcal{H} \subseteq \mathbb{R}^{q}$ and $\lambda(t) \in \mathbb{R}_{+}^{m}$, and $\overline{\|u(t)\|}=\lim _{T \rightarrow \infty} \frac{1}{T} \int_{0}^{T}\|u(t)\| d t=\beta<\frac{a_{3}}{a_{4} L_{x}}$. Then the average tracking error $\overline{\left\|\mathrm{x}_{e}\right\|^{2}}$ of the proposed algorithm 34-35) converges to 0 .

Corollary 3 can be obtained from Theorem 4 in a straight-forward manner by replacing the variable $\widetilde{\mathbf{z}}_{e}$ with $\mathbf{z}_{e}$. 


\section{B. Distributive Implementation}

The proposed algorithm (34)-(35) discussed in the previous section requires centralized computation for the compensation term $\widehat{\varphi}(\widetilde{\mathbf{x}} ; \mathbf{h}(t))$. However, for a wireless network, distributive solutions are usually desired. One way for distributive implementation is via message passing (i.e. communicating with other nodes to obtain non-local variables), while each node computes a version of $\widehat{\varphi}(\widetilde{\mathbf{x}} ; \mathbf{h}(t))$ based on the global knowledge. However, this involves a significant communication overhead and consumes a lot of computing resources. In the following, we propose a low complexity distributive implementation for computing the compensation term.

Consider an ad hoc network topology as depicted in Problem 2 in Section III-B2. To facilitate distributive implementation of the compensated primal-dual algorithm (34)-(35), we partition the whole network into $G$ groups of nodes and partition the collection of optimization variables $\widetilde{\mathbf{x}}(t)=$ $\left[\widetilde{\mathbf{x}}_{1}(t)\left|\widetilde{\mathbf{x}}_{2}(t)\right| \ldots \mid \widetilde{\mathbf{x}}_{G}(t)\right]$ accordingly. Let $B(t)=\frac{\partial}{\partial \widetilde{\mathbf{x}}} F(\widetilde{\mathbf{x}}(t) ; \mathbf{h}(t))$ and $K(t)=\frac{\partial}{\partial \mathbf{h}} F(\widetilde{\mathbf{x}}(t) ; \mathbf{h}(t))$. We then impose a block diagonal structure to the matrix $B(t)$ and partition $K(t)$ as

$$
\widehat{B}(t)=\operatorname{blkdiag}\left\{B_{\widetilde{\mathbf{x}}_{1}}(t), B_{\widetilde{\mathbf{x}}_{2}}(t), \ldots, B_{\widetilde{\mathbf{x}}_{G}}(t)\right\}, \quad \widehat{K}(t)^{T}=\left[K_{\widetilde{\mathbf{x}}_{1}}^{T}(t)\left|K_{\widetilde{\mathbf{x}}_{2}}^{T}(t)\right| \ldots \mid K_{\widetilde{\mathbf{x}}_{G}}^{T}(t)\right]
$$

where $\widetilde{\mathbf{x}}_{i}(t), \forall i=1, \ldots, G$ is the collection of variables for the $i$-th group of nodes, $B_{\widetilde{\mathbf{x}}_{i}}(t)=$ $\frac{\partial}{\partial \widetilde{\mathbf{x}}_{i}} F_{\widetilde{\mathbf{x}}_{i}}(\widetilde{\mathbf{x}}(t) ; \mathbf{h}(t))$ is a block of $B(t)$ for the corresponding variables $\widetilde{\mathbf{x}}_{i}(t)$, and $K_{\widetilde{\mathbf{x}}_{i}}(t)=\frac{\partial}{\partial \mathbf{h}} F_{\widetilde{\mathbf{x}}_{i}}(\widetilde{\mathbf{x}}(t) ; \mathbf{h}(t))$ is a block of $K(t)$ for the corresponding variables $\widetilde{\mathbf{x}}_{i}(t)$. Therefore, the compensation term for the variable $\widetilde{\mathbf{x}}_{i}(t)$ is just $\widehat{\varphi}_{\widetilde{\mathbf{x}}_{i}}(t)=-B_{\widetilde{\mathbf{x}}_{i}}^{-1}(t) K_{\widetilde{\mathbf{x}}_{i}}(t)$. For example, for a NUM problem with a topology depicted in Fig. 2, the network can be partitioned into two parts according to the two links, where $\widetilde{\mathbf{x}}_{1}=\left\{r_{12}, r_{13}, \boldsymbol{\lambda}_{1}\right\}$ and $\widetilde{\mathbf{x}}_{2}=\left\{r_{23}, \boldsymbol{\lambda}_{2}\right\}$ (i.e. $\boldsymbol{\lambda}_{i}$ are the corresponding dual variables). The distributive update equation for the collection of variable $\widetilde{\mathbf{x}}_{i}$ is

$$
\dot{\widetilde{\mathbf{x}}}_{i}=\left[\left(\nabla_{\widetilde{\mathbf{x}}_{i}} \mathcal{L}(\widetilde{\mathbf{x}} ; \mathbf{h}(t))+\widehat{\varphi}_{\widetilde{\mathbf{x}}_{i}}(\widetilde{\mathbf{x}} ; \mathbf{h}(t))\right)^{T}\right]_{\widetilde{\mathbf{x}}_{i}}^{+} \quad \forall i=1, \ldots, G
$$

where $\nabla_{\widetilde{\mathbf{x}}_{i}} \mathcal{L}(\cdot)$ denotes the searching direction by the gradient of $\frac{\partial \mathcal{L}(\cdot)}{\partial \widetilde{\mathbf{x}}_{i}}$ and $[\cdot]_{\widetilde{\mathbf{x}}_{i}}^{+}$denotes the corresponding projection for the variable $\widetilde{\mathbf{x}}_{i}$.

As the terms $B_{\widetilde{\mathbf{x}}_{i}}(t)$ and $K_{\widetilde{\mathbf{x}}_{i}}(t)$ just involve local variables, $\widehat{\varphi}_{\widetilde{\mathbf{x}}_{i}}(t)$ can be computed locally using only local information and hence the update equation (38) can be implemented distributively. Note that in wireless communication networks, nodes that are far away from each others usually have weak connections, and hence $B(t)$ is actually a sparse matrix with dense diagonal entries. Therefore, using the block diagonal version $\widehat{B}(t)$ in 37 will not contributes too much performance loss in the proposed algorithm.

\section{RESULTS AND DisCUSSIONS}

In this section, we shall simulate the tracking performance of the primal-dual algorithms for various saddle point problems we studied before. We evaluate the performance of the proposed algorithms 
with the adaptive compensation term compared with the baseline algorithms in [3], [20], [21]. The system performance loss due to the time varying channels and the tracking errors is also illustrated.

\section{A. Convergence Performance of the Strongly Concave-convex Saddle Point Problem and the Degraded}

\section{Saddle Point Problem}

The simulations of the convergence performance for the strongly concave-convex saddle point problem and the degraded saddle point problem are based on the examples described in Section III-B and Section IV-B, respectively. The average tracking error in the jamming problem is defined as $\overline{e^{2}}=\mathbb{E}\left[\left\|\widetilde{\mathbf{x}}(t)-\widetilde{\mathbf{x}}^{*}(t)\right\|^{2}\right]$ where $\widetilde{\mathbf{x}}(t)=\operatorname{vec}([Q(t) Z(t)])$, while $\overline{e^{2}}=\mathbb{E}\left[\left\|\mathbf{r}(t)-\mathbf{r}^{*}(t)\right\|^{2}\right]$ defines the average tracking error in the NUM problem, where $\mathbf{r}(t)$ is the rate allocation variables. In both cases, the CSI model is specified by an AR process $\dot{\mathbf{h}}=-a(\mathbf{h}(t)-\overline{\mathbf{h}})+\sqrt{2 a} w(t)$, where $\overline{\mathbf{h}}=\mathbf{1}$, $w(t)$ is a zero-mean unity-variance Gaussian process and $a$ is a parameter to synthesize the fading rate. Fig. 7 and Fig 8 illustrate the average tracking errors $\overline{e^{2}}$ versus the fading rate parameter $a$ in both of the problems. As $a$ increases, the CSI changes more rapidly and the tracking errors of the primal dual algorithm increase. Moreover, for the same parameter $a$, the larger the average receiving SNR is, the larger tracking error will be.

\section{B. Convergence Performance of the Proposed Algorithm with Compensation}

To evaluate the algorithm performance in a more concrete communication scenario, we consider a NUM problem of a wireless ad hoc network with 6 nodes 8 links and 8 data flows as illustrated in Fig 6. The data flows are delivered simultaneously with fixed routes. Links from the same transmitting node occupy different subbands while the interference at each receiving node is handled by multiuser detection (MUD) techniques. The problem is to optimize the transmit power under the time varying channels to support required traffic data rates. Similar ad hoc network scenario is also considered in [22]. Similarly, the CSI model is specified by the AR process $\dot{\mathbf{h}}=-a(\mathbf{h}(t)-\overline{\mathbf{h}})+\sqrt{2 a} w(t)$, where $a$ is a parameter to determine the CSI fading rate.

The proposed algorithms with adaptive compensations are compared with the following baseline algorithms.

- Baseline 1 - Conventional Primal-Dual Gradient Algorithm (ConPDGA) [1], [3]: The primal and dual variables are updated simultaneously in (2)-(3) in the gradients of the objective function.

- Baseline 2 - Averaging Primal-Dual Gradient Algorithm (AvgPDGA) [20]: The primal and dual variables are updated in the gradients of the objective function, and the approximate primal solutions are generated by averaging over the past primal solutions.

- Baseline 3 - Perturbed Primal-Dual Gradient Algorithm (PerPDGA) [21], [23]: The primal and dual variables are updated in the gradients evaluated at perturbed points that are generated 
from current points via auxiliary mappings.

- Proposed Primal-Dual Algorithm - Centralized: The primal and dual variables are updated in a compensated direction in (34)- (35) to offset the potential movement of the saddle point.

- Proposed Primal-Dual Algorithm - Distributed: The primal and dual variables are updated in a compensated direction in (38) with imposed block diagonal structure in (37).

Fig. 9 shows the comparison of the average tracking error $\overline{e^{2}}$ versus $a$ in the time varying CSI model. The tracking errors have been reduced greatly after introducing the compensation terms in both of the proposed algorithms.

\section{Performance Loss due to Tracking Errors}

In this section, we consider the degradation of the system performance (network throughput) and the CSI fading rate. Due to the time varying channels, the problem constraints (e.g. the link capacity constraints) may not be satisfied at every time slot. As a result, packet drops may occur and we define the system performance as the average actual throughput given by:

$$
\bar{R}=\mathbb{E}\left[\sum_{k \in \mathcal{K}}\left(\sum_{(s, d) \in \mathcal{T}(k)} r_{s d}\right) \times 1\left\{\tilde{\mathbf{c}}_{k} \in \mathcal{C}_{k}(\mathbf{p} ; \mathbf{h})\right\}\right]
$$

where $\mathcal{K}$ denotes the set of receiving nodes, $\mathcal{T}(k)$ denotes the set of traffic flows arrived at node $k, \tilde{\mathbf{c}}_{k}$ denotes the vector of link transmission rates of the links towards node $k, \mathcal{C}_{k}(\cdot)$ denotes the capacity region at receiving node $k$ and $1($.$) is the indicator function to account for the penalty of$ constraint violation due to time varying CSI. For example, at node $k=5$ in Fig. 6, the set of traffic flows arrived is $\mathcal{T}(5)=\left\{r_{22}, r_{42}, r_{11}\right\}$ and $\tilde{\mathbf{c}}_{5}=\left(\tilde{r}_{5}, \tilde{r}_{7}\right)$ is a rate vector corresponding to transmission rates $\tilde{r}_{5}$ and $\tilde{r}_{7}$ allocated to link 5 and link 7 , respectively.

Fig 10 shows the the average network throughput versus the channel fading rate $a$. The average throughput decreases when the fading rate $a$ increases, as indicated by a growing average tracking error in Fig 9 . The results also show that the proposed algorithms significantly outperform over all the other baselines.

\section{CONCLUSIONS}

In this paper, we have analyzed the convergence behavior of the primal-dual algorithm for solving a saddle point problem under time varying CSI, which was modeled as an AR process. The convergence results have been derived by studying the stabilities of the equivalent virtual dynamic systems based on the Lyapunov theory from the control theoretical approach. We showed that for both the strongly concave-convex saddle point problem and the degraded saddle point problem, the average tracking errors were given by $\mathcal{O}\left(\alpha^{2}\right)$, where $\alpha^{2}$ represents the average power of the exogenous excitation 
induced to the CSI dynamics. Based on these analyses, we have proposed a novel adaptive primaldual algorithm with a predictive compensation to counteract the effects of the time varying CSI. We showed that the average tracking error of the proposed algorithm converges to zero despite time varying CSI. Numerical results were consistent with our analysis and the proposed algorithm demonstrated significantly better convergence performance over the baseline schemes.

\section{APPENDIX A}

\section{PROOF OF THEOREM 1}

Proof: The equilibrium point $\widetilde{\mathbf{x}}^{*}(\mathbf{h}(t))$ of the virtual dynamic system $\widetilde{\mathcal{X}}$ in 11 satisfies $\widetilde{f}\left(\widetilde{\mathbf{x}}^{*}(\mathbf{h}(t)) ; \mathbf{h}(t)\right)=$ 0 . By Definition 1, it is equivalent to

$$
\frac{\partial}{\partial \mathbf{x}} \mathcal{L}\left(\mathbf{x}^{*}, \lambda^{*} ; \mathbf{h}(t)\right)=0, \quad \lambda_{i} \frac{\partial}{\partial \lambda_{i}} \mathcal{L}\left(\mathbf{x}^{*}, \lambda^{*} ; \mathbf{h}(t)\right)=0, \forall i
$$

which is the optimality condition for the saddle point problem. As the saddle point problem (1) is strongly concave in $\mathbf{x}$, the saddle point $\left[\mathbf{x}^{*}(\mathbf{h}(t)), \lambda^{*}(\mathbf{h}(t))\right]$ is uniquely determined by [39], which proofs that the saddle point and equilibrium are identical.

From Definition 1, the virtual dynamic system (11) and the primal-dual algorithm (2)-(3) share the same formulation. Hence the trajectories of the two are the same.

\section{APPENDIX B}

\section{Proof of Lemma 1}

Proof: Take $V\left(\widetilde{\mathbf{x}}_{e}\right)=\frac{1}{2 \kappa} \widetilde{\mathbf{x}}_{e}^{T} \widetilde{\mathbf{x}}_{e}$ as a Lyapunov function defined along the trajectory of the virtual dynamic system (12). Suppose that $\widetilde{\mathbf{x}}_{e}+\widetilde{\mathbf{x}}^{*}$ is in the interior of the domain $\mathbb{R}^{n} \times \mathbb{R}_{+}^{m}$. From

$$
\nabla \widetilde{f}_{e}\left(\widetilde{\mathbf{x}}_{e} ; \mathbf{h}\right)=\kappa\left[\begin{array}{cc}
\nabla_{\mathbf{x}}^{2} \mathcal{L} & \nabla_{\mathbf{x} \lambda} \mathcal{L} \\
-\nabla_{\mathbf{x} \lambda}^{T} \mathcal{L} & -\nabla_{\lambda}^{2} \mathcal{L}
\end{array}\right]
$$

and the strong convexity / concavity properties $\nabla_{\mathbf{x}}^{2} \mathcal{L} \preceq-M_{x} I$ and $\nabla_{\lambda}^{2} \mathcal{L} \succeq M_{\lambda} I$, we obtain

$$
\widetilde{\mathbf{x}}_{e}^{T} \nabla \widetilde{f}_{e}\left(\widetilde{\mathbf{x}}_{e} ; \mathbf{h}\right) \widetilde{\mathbf{x}}_{e} \leq-\kappa \min \left\{M_{x}, M_{\lambda}\right\}\left\|\widetilde{\mathbf{x}}_{e}\right\|^{2} \triangleq-\kappa M\left\|\widetilde{\mathbf{x}}_{e}\right\|^{2}
$$

Then $\dot{V}(\mathbf{0})=0$ and for any $\widetilde{\mathbf{x}}_{e} \neq 0$,

$$
\begin{aligned}
\dot{V}\left(\widetilde{\mathbf{x}}_{e}\right) & =\nabla V\left(\widetilde{\mathbf{x}}_{e}\right) \dot{\tilde{\mathbf{x}}}_{e}=\frac{1}{\kappa} \widetilde{\mathbf{x}}_{e}^{T} \dot{\tilde{\mathbf{x}}}_{e}=\frac{1}{\kappa} \widetilde{\mathbf{x}}_{e}^{T} \widetilde{f}_{e}\left(\widetilde{\mathbf{x}}_{e} ; \mathbf{h}\right) \\
& =\frac{1}{\kappa} \widetilde{\mathbf{x}}_{e}^{T} \widetilde{f}_{e}\left(\mathbf{0}_{+} ; \mathbf{h}\right)+\frac{1}{\kappa} \widetilde{\mathbf{x}}_{e}^{T} \int_{0}^{1} \nabla \widetilde{f}_{e}\left(\xi \widetilde{\mathbf{x}}_{e} ; \mathbf{h}\right) d \xi \widetilde{\mathbf{x}}_{e} \\
& \leq \frac{1}{\kappa} \widetilde{\mathbf{x}}_{e}^{T} \widetilde{f}_{e}\left(\mathbf{0}_{+} ; \mathbf{h}\right)-\int_{0}^{1} \frac{1}{\xi} M d\left\|\xi \widetilde{\mathbf{x}}_{e}\right\|^{2} \\
& \leq-2 M\left\|\widetilde{\mathbf{x}}_{e}\right\|^{2}
\end{aligned}
$$

where $\widetilde{\mathbf{x}}_{e}^{T} \widetilde{f}_{e}\left(\mathbf{0}_{+} ; \mathbf{h}\right)=<\widetilde{\mathbf{x}}-\widetilde{\mathbf{x}}^{*}, \nabla \overline{\mathcal{L}}\left(\widetilde{\mathbf{x}}^{*} ; \mathbf{h}\right)>\leq 0$ from the optimality condition of the original saddle point problem. Here for simplicity, we denote $\nabla \overline{\mathcal{L}}(\widetilde{\mathbf{x}} ; \mathbf{h})=\left[\frac{\partial}{\partial \mathbf{x}} \mathcal{L}(\mathbf{x}, \lambda ; \mathbf{h})-\frac{\partial}{\partial \lambda} \mathcal{L}(\mathbf{x}, \lambda ; \mathbf{h})\right]^{T}$. 
Therefore, using the Lyapunov theory [9], we conclude that the virtual dynamic system is exponentially stable for every time-invariant $\mathbf{h}$.

\section{APPENDIX C}

\section{PROOF OF LEMMA 2}

Proof: One possible choice of Lyapunov function on virtual state $\widetilde{\mathbf{z}}_{e}$ along the algorithm trajectory is $V\left(\widetilde{\mathbf{z}}_{e}\right)=\frac{1}{2 \kappa} \widetilde{\mathbf{x}}_{e}^{T} \widetilde{\mathbf{x}}_{e}+\frac{1}{2} \mathbf{h}_{e}^{T} \mathbf{h}_{e}$. Then we have

$$
\min \left\{\frac{1}{2 \kappa}, \frac{1}{2}\right\}\left\|\widetilde{\mathbf{z}}_{e}\right\|^{2} \leq V\left(\widetilde{\mathbf{z}}_{e}\right)=\frac{1}{2 \kappa}\left\|\widetilde{\mathbf{x}}_{e}\right\|^{2}+\frac{1}{2}\left\|\mathbf{h}_{e}\right\|^{2} \leq \max \left\{\frac{1}{2 \kappa}, \frac{1}{2}\right\}\left\|\widetilde{\mathbf{z}}_{e}\right\|^{2}
$$

and

$$
\begin{aligned}
\dot{V}\left(\widetilde{\mathbf{z}}_{e}\right) & =\frac{\partial V}{\partial \widetilde{\mathbf{z}}_{e}} Z\left(\widetilde{\mathbf{z}}_{e}\right)=\left[\begin{array}{cc}
\frac{1}{\kappa} \widetilde{\mathbf{x}}_{e}^{T} & \mathbf{h}_{e}^{T}
\end{array}\right]\left[\begin{array}{c}
\widetilde{f}_{e}\left(\widetilde{\mathbf{x}}_{e} ; \mathbf{h}_{e}+\overline{\mathbf{h}}\right)+\varphi\left(\mathbf{h}_{e}+\overline{\mathbf{h}}\right) A \mathbf{h}_{e} \\
A \mathbf{h}_{e}
\end{array}\right] \\
& =\frac{1}{\kappa} \widetilde{\mathbf{x}}_{e} \widetilde{f}_{e}\left(\widetilde{\mathbf{x}}_{e} ; \mathbf{h}\right)+\mathbf{h}^{T} A \mathbf{h}+\frac{1}{\kappa} \widetilde{\mathbf{x}}_{e} \varphi(\mathbf{h}) A \mathbf{h} \\
& \leq-2 M\left\|\widetilde{\mathbf{x}}_{e}\right\|^{2}+\lambda_{\max }(A)\|\mathbf{h}\|^{2}+\frac{1}{\kappa}\left\|\widetilde{\mathbf{x}}_{e}\right\|\|\varphi(\mathbf{h}) A\|\|\mathbf{h}\| \\
& \leq-\left[\min \left\{2 M,-\lambda_{\max }(A)\right\}-\frac{1}{\kappa}\|\varphi(\mathbf{h}) A\|\right]\left\|\widetilde{\mathbf{z}}_{e}\right\|^{2} \\
& =-a_{3}\left\|\widetilde{\mathbf{z}}_{e}\right\|^{2}
\end{aligned}
$$

where $a_{3} \triangleq \min \left\{2 M,-\lambda_{\max }(A)\right\}-\frac{1}{\kappa}\|\varphi(\mathbf{h}) A\|>0$ by the condition in (15). In addition, we have

$$
\left\|\frac{\partial V}{\partial \widetilde{\mathbf{z}}_{e}}\right\|=\left\|\left[\begin{array}{cc}
\frac{1}{\kappa} \widetilde{\mathbf{x}}_{e}^{T} & \mathbf{h}^{T}
\end{array}\right]\right\| \leq \sqrt{\frac{1}{\kappa^{2}}\left\|\widetilde{\mathbf{x}}_{e}\right\|^{2}+\|\mathbf{h}\|^{2}} \leq \max \left\{\frac{1}{\kappa}, 1\right\}\left\|\widetilde{\mathbf{z}}_{e}\right\|
$$

Therefore, using Lyapunov theory [9], we conclude that the joint system is exponentially stable and (16)-(18) are satisfied with $a_{1}=\min \{1 / 2 \kappa, 1 / 2\}, a_{2}=\max \{1 / 2 \kappa, 1 / 2\}, a_{3}=\min \left\{2 M,-\lambda_{\max }(A)\right\}-$ $\frac{1}{\kappa}\|\varphi(\mathbf{h}) A\|$ and $a_{4}=\max \{1 / \kappa, 1\}$.

\section{APPENDIX D}

\section{PROOF OF THEOREM 2}

Proof: From Lemma 2, there exists a Lyapunov function $V\left(\widetilde{\mathbf{z}}_{e}\right)$ defined on the joint state $\widetilde{\mathbf{z}}_{e}$ along the trajectory $\dot{\mathbf{z}}_{e}=\widetilde{\mathcal{Z}}\left(\widetilde{\mathbf{z}}_{e}\right)$, satisfying $(16)-(18)$. Then, for the system $(19)$,

$$
\dot{V}\left(\widetilde{\mathbf{z}}_{e}\right)=\frac{\partial V}{\partial \widetilde{\mathbf{z}}_{e}} \widetilde{\mathcal{Z}}\left(\widetilde{\mathbf{z}}_{e}\right)+\frac{\partial V}{\partial \widetilde{\mathbf{z}}_{e}} \Phi\left(\mathbf{h}_{e}\right) u(t) \leq-a_{3}\left\|\widetilde{\mathbf{z}}_{e}\right\|^{2}+a_{4}\left\|\Phi\left(\mathbf{h}_{e}\right)\right\|\left\|\widetilde{\mathbf{z}}_{e}\right\|\|u(t)\|
$$

Suppose there exists $\epsilon>0$, such that

$$
\lim _{T \rightarrow \infty} \frac{1}{T} \int_{0}^{T}\left\|\widetilde{\mathbf{z}}_{e}(\tau)\right\|^{2} d \tau \geq \frac{a_{4}^{2}}{a_{3}^{2}} \gamma^{2} \alpha^{2}+\epsilon
$$

Then given any $t>0$, there exists $T_{0}(t)>0$, such that for all $T>T_{0}$,

$$
\frac{1}{t+T} \int_{0}^{t+T}\left\|\widetilde{\mathbf{z}}_{e}(\tau)\right\|^{2} d \tau \geq \frac{a_{4}^{2}}{a_{3}^{2}} \gamma^{2} \alpha^{2}+\frac{\epsilon}{2}
$$


and there exists $T_{1}(t)>0$, such that for all $T>T_{1}$,

$$
\frac{1}{t+T} \int_{0}^{t}\left\|\widetilde{\mathbf{z}}_{e}(\tau)\right\|^{2} d \tau \leq \frac{\epsilon}{4}
$$

Choose $0<\eta<\frac{\epsilon}{4} \frac{a_{3}^{2}}{a_{4}^{2} \gamma^{2}}$, and then from $\overline{\|u(t)\|^{2}}=\lim _{T \rightarrow \infty} \frac{1}{T} \int_{0}^{T}\|u(t)\|^{2} d t \leq \alpha^{2}$, there exists $T_{2}(t)>0$, such that for all $T>T_{2}$,

$$
\frac{1}{T} \int_{t}^{t+T}\|u(\tau)\|^{2} d \tau=\frac{t+T}{T} \frac{1}{t+T} \int_{0}^{t+T}\|u(\tau)\|^{2} d \tau \leq \frac{t+T}{T}\left(\alpha^{2}+\eta\right)
$$

Finally, let $T_{3}(t)=\max \left\{T_{0}(t), T_{1}(t), T_{2}(t)\right\}$. Then for all $T>T_{3}$,

$$
\frac{1}{T} \int_{t}^{t+T}\left\|\widetilde{\mathbf{z}}_{e}(\tau)\right\|^{2} d \tau=\frac{t+T}{T}\left[\frac{1}{t+T}\left(\int_{0}^{t+T}\left\|\widetilde{\mathbf{z}}_{e}(\tau)\right\|^{2} d \tau-\int_{0}^{t}\left\|\widetilde{\mathbf{z}}_{e}(\tau)\right\|^{2} d \tau\right)\right] \geq \frac{t+T}{T}\left(\frac{a_{4}^{2}}{a_{3}^{2}} \gamma^{2} \alpha^{2}+\frac{\epsilon}{4}\right)
$$

Hence the $T$-step Lyapunov drift is given by

$$
\begin{aligned}
& V\left(\widetilde{\mathbf{z}}_{e}(t+T)\right)-V\left(\widetilde{\mathbf{z}}_{e}(t)\right) \\
\leq & -\int_{t}^{t+T} a_{3}\left\|\widetilde{\mathbf{z}}_{e}(\tau)\right\|^{2} d \tau+\int_{t}^{t+T} a_{4} \gamma\left\|\widetilde{\mathbf{z}}_{e}(\tau)\right\|\|u(\tau)\| d \tau \\
\leq & -a_{3} \int_{t}^{t+T}\left\|\widetilde{\mathbf{z}}_{e}(\tau)\right\|^{2} d \tau+\sqrt{\int_{t}^{t+T} a_{4}^{2} \gamma^{2}\left\|\widetilde{\mathbf{z}}_{e}(\tau)\right\|^{2} d \tau} \cdot \sqrt{\int_{t}^{t+T}\|u(\tau)\|^{2} d \tau} \\
= & \left(-a_{3} \sqrt{\int_{t}^{t+T}\left\|\widetilde{\mathbf{z}}_{e}(\tau)\right\|^{2} d \tau}+a_{4} \gamma \sqrt{\int_{t}^{t+T}\|u(\tau)\|^{2} d \tau}\right) \sqrt{\int_{t}^{t+T}\left\|\widetilde{\mathbf{z}}_{e}(\tau)\right\|^{2} d t \tau} \\
\leq & \left(-a_{3} \sqrt{\left(\frac{a_{4}^{2}}{a_{3}^{2}} \gamma^{2} \alpha^{2}+\frac{\epsilon}{4}\right)(t+T)}+a_{4} \gamma \sqrt{\left(\alpha^{2}+\eta\right)(t+T)}\right) \sqrt{\int_{t}^{t+T}\left\|\widetilde{\mathbf{z}}_{e}(\tau)\right\|^{2} d t \tau} \\
< & 0
\end{aligned}
$$

by (41) and (42). By the property of the Lyapunov function $V\left(\widetilde{\mathbf{z}}_{e}\right)$ in 16 , for any given $t>0$, we have $\left\|\widetilde{\mathbf{z}}_{e}(t+T)\right\|<\left\|\widetilde{\mathbf{z}}_{e}(t)\right\|$ for all $T>T_{3}$. Hence, we have $\lim _{t \rightarrow \infty}\left\|\widetilde{\mathbf{z}}_{e}(t)\right\|=0$, which contradicts to the assumption in $\left(40\right.$. So the average trajectory $\overline{\left\|\widetilde{\mathbf{z}}_{e}\right\|^{2}} \leq \frac{a_{4}^{2}}{a_{3}^{2}} \gamma^{2} \alpha^{2}$, which proofs the stability.

\section{APPENDIX E}

\section{PROOF OF LEMMA 4}

Proof: A simple choice of Lyapunov function is $V\left(\mathbf{z}_{e}\right)=V_{0}\left(\mathbf{x}_{e}\right)+\frac{1}{2} \mathbf{h}_{e}^{T} \mathbf{h}_{e}$, where $V_{0}\left(\mathbf{x}_{e}\right)=$ $\left.V_{x}\left(\widetilde{\mathbf{x}}_{e}\right)\right|_{\lambda_{e}=0}=\frac{1}{2 \kappa} \mathbf{x}_{e}^{T} \mathbf{x}_{e}$ and $\frac{\partial V_{0}\left(\mathbf{x}_{e}\right)}{\partial \widetilde{\mathbf{x}}_{e}}=\frac{1}{\kappa}\left[\mathbf{x}_{e}^{T} \mathbf{0}^{T}\right]$. Hence we have

$$
\min \left\{\frac{1}{2 \kappa}, \frac{1}{2}\right\}\left\|\mathbf{z}_{e}\right\|^{2} \leq c_{1}\left\|\mathbf{x}_{e}\right\|^{2}+\frac{1}{2}\|\mathbf{h}\|^{2} \leq V\left(\mathbf{z}_{e}\right) \leq c_{2}\left\|\mathbf{x}_{e}\right\|^{2}+\frac{1}{2}\|\mathbf{h}\|^{2} \leq \max \left\{\frac{1}{2 \kappa}, \frac{1}{2}\right\}\left\|\mathbf{z}_{e}\right\|^{2}
$$


and

$$
\begin{aligned}
\frac{d V\left(\mathbf{z}_{e}\right)}{d t} & =\frac{\partial V\left(\mathbf{z}_{e}\right)}{\partial \widetilde{\mathbf{z}}_{e}} \frac{d \widetilde{\mathbf{z}}_{e}}{d t}=\left[\begin{array}{ll}
\frac{\partial V_{0}\left(\mathbf{x}_{e}\right)}{\partial \mathbf{x}_{e}} & \mathbf{h}_{e}^{T}
\end{array}\right]\left[\begin{array}{c}
\widetilde{f}_{e}\left(\widetilde{\mathbf{x}}_{e} ; \mathbf{h}_{e}+\overline{\mathbf{h}}\right)+\varphi\left(\mathbf{h}_{e}+\overline{\mathbf{h}}\right) A \mathbf{h}_{e} \\
A \mathbf{h}_{e}
\end{array}\right] \\
& =\frac{\partial V_{0}\left(\mathbf{x}_{e}\right)}{\partial \widetilde{\mathbf{x}}_{e}} \widetilde{f}_{e}\left(\widetilde{\mathbf{x}}_{e} ; \mathbf{h}_{e}+\overline{\mathbf{h}}\right)+\frac{\partial V_{0}\left(\mathbf{x}_{e}\right)}{\partial \widetilde{\mathbf{x}}_{e}} A \mathbf{h}_{e}+\mathbf{h}_{e}^{T} A \mathbf{h}_{e} \\
& \leq-c_{3}\left\|\mathbf{x}_{e}\right\|^{2}+c_{4}\left\|\mathbf{x}_{e}\right\|\left\|\varphi_{x}(\mathbf{h}) A\right\|\left\|\mathbf{h}_{e}\right\|+\lambda_{\max }(A)\left\|\mathbf{h}_{e}\right\|^{2} \\
& \leq-\left[\min \left\{c_{3},-\lambda_{\max }(A)\right\}-c_{4}\left\|\varphi_{x}(\mathbf{h}) A\right\|\right]\left\|\mathbf{z}_{e}\right\|^{2} \\
& \triangleq-a_{3}\left\|\mathbf{z}_{e}\right\|^{2}
\end{aligned}
$$

where $c_{3}=2 M_{x}$ and $c_{4}=1 / \kappa$ following the discussion in Lemma 3. Moreover,

$$
\left\|\frac{\partial V\left(\mathbf{z}_{e}\right)}{\partial \mathbf{z}_{e}}\right\|=\left\|\left[\begin{array}{ll}
\frac{\partial V_{0}\left(\mathbf{x}_{e}\right)}{\partial \mathbf{x}_{e}} & \mathbf{h}^{T}
\end{array}\right]\right\| \leq \sqrt{c_{4}^{2}\left\|\mathbf{x}_{e}\right\|^{2}+\|\mathbf{h}\|^{2}} \leq \sqrt{\max \left\{\frac{1}{\kappa^{2}}, 1\right\}}\left\|\mathbf{z}_{e}\right\|
$$

Therefore, using the Lyapunov theory [9], we conclude that the system $\dot{\mathbf{z}}_{e}=\mathcal{Z}\left(\mathbf{z}_{e}\right)$ is exponentially stable for the joint state $\mathbf{z}_{e}=\left(\mathbf{x}_{e}, \mathbf{h}\right)$ and 27)-(29) are satisfied with $a_{1}=\min \{1 /(2 \kappa), 1 / 2\}$, $a_{2}=\max \{1 /(2 \kappa), 1 / 2\}, a_{3}=\min \left\{2 M_{x},-\lambda_{\max }(A)\right\}-\frac{1}{\kappa}\left\|\varphi_{x}(\mathbf{h}) A\right\|$ and $a_{4}=\sqrt{\max \left\{1 / \kappa^{2}, 1\right\}}$.

\section{APPENDIX F}

\section{PROOF OF THEOREM 4}

Proof: Consider the virtual dynamic system with compensation in (31). Since

$$
\Phi\left(\mathbf{h}_{e}\right)-\widehat{\Phi}\left(\widetilde{\mathbf{z}}_{e}\right)=\left[\begin{array}{c}
\widehat{\varphi}\left(\widetilde{\mathbf{x}}^{*} ; \mathbf{h}(t)\right)-\widehat{\varphi}(\widetilde{\mathbf{x}} ; \mathbf{h}(t)) \\
0
\end{array}\right]
$$

then $\left\|\Phi\left(\mathbf{h}_{e}\right)-\widehat{\Phi}\left(\widetilde{\mathbf{z}}_{e}\right)\right\|=\left\|\widehat{\varphi}\left(\widetilde{\mathbf{x}}^{*} ; \mathbf{h}(t)\right)-\widehat{\varphi}(\widetilde{\mathbf{x}} ; \mathbf{h}(t))\right\| \leq L\left\|\widetilde{\mathbf{x}}_{e}\right\| \leq L\left\|\widetilde{\mathbf{z}}_{e}\right\|$. Applying the Lyapunov function $V\left(\widetilde{\mathbf{z}}_{e}\right)$ in Lemma 2 to the virtual dynamic system $\widetilde{\mathcal{Z}}_{e}(\widehat{u})$, we obtain

$$
\begin{aligned}
\dot{V}\left(\widetilde{\mathbf{z}}_{e}\right) & =\frac{\partial V}{\partial \widetilde{\mathbf{z}}_{e}} \widetilde{\mathcal{Z}}\left(\widetilde{\mathbf{z}}_{e}\right)+\frac{\partial V}{\partial \widetilde{\mathbf{z}}_{e}} \Phi\left(\mathbf{h}_{e}\right) u(t) \leq-a_{3}\left\|\widetilde{\mathbf{z}}_{e}\right\|^{2}+a_{4}\left\|\Phi\left(\mathbf{h}_{e}\right)-\widehat{\Phi}\left(\widetilde{\mathbf{z}}_{e}\right)\right\|\|u(t)\|\left\|\widetilde{\mathbf{z}}_{e}\right\| \\
& \leq-a_{3}\left\|\widetilde{\mathbf{z}}_{e}\right\|^{2}+a_{4} L\|u(t)\|\left\|\widetilde{\mathbf{z}}_{e}\right\|^{2}
\end{aligned}
$$

Suppose there exists $\epsilon>0$, such that

$$
\lim _{T \rightarrow \infty} \frac{1}{T} \int_{t}^{t+T}\left\|\widetilde{\mathbf{z}}_{e}(\tau)\right\|^{2} d \tau>\epsilon
$$

Then there exists $T_{0}(t)>0$, such that for all $T>T_{0}, \frac{1}{T} \int_{t}^{t+T}\left\|\widetilde{\mathbf{z}}_{e}(\tau)\right\|^{2} d \tau>\frac{\epsilon}{2}$.

As $\lim _{T \rightarrow \infty} \frac{1}{T} \int_{t}^{t+T}\|u(\tau)\| d \tau=\beta<\frac{a_{3}}{a_{4} L}$, we choose $\eta=\left(\frac{a_{3}}{a_{4} L}-\beta\right) / 2$. Then there exists $T_{1}(t)>0$, such that for all $T>T_{1}, \frac{1}{T} \int_{t}^{t+T}\|u(\tau)\| d \tau<\beta+\eta<\frac{a_{3}}{a_{4} L}$.

Observed that, $\widetilde{\mathbf{z}}_{e}(t)$ only depends on all the realizations before $u\left(t_{-}\right)$. Therefore $\|u(t)\|$ and $\left\|\widetilde{\mathbf{z}}_{e}(t)\right\|^{2}$ are independent and hence,

$$
\lim _{T \rightarrow \infty} \frac{1}{T} \int_{t}^{t+T}\|u(\tau)\|\left\|\widetilde{\mathbf{z}}_{e}(\tau)\right\|^{2} d \tau=\lim _{T \rightarrow \infty} \frac{1}{T} \int_{t}^{t+T}\|u(\tau)\| d \tau \cdot \frac{1}{T} \int_{t}^{t+T}\left\|\widetilde{\mathbf{z}}_{e}(\tau)\right\|^{2} d \tau .
$$


Therefore, if we choose any $0<\delta<\eta \epsilon / 2$, then there exists $T_{2}(t)>0$, such that for all $T>T_{2}$,

$$
\frac{1}{T} \int_{t}^{t+T}\|u(\tau)\|\left\|\widetilde{\mathbf{z}}_{e}(\tau)\right\|^{2} d \tau \leq \frac{1}{T} \int_{t}^{t+T}\|u(\tau)\| d \tau \cdot \frac{1}{T} \int_{t}^{t+T}\left\|\widetilde{\mathbf{z}}_{e}(\tau)\right\|^{2} d \tau+\delta
$$

As a result, let $T_{3}(t)=\max \left\{T_{0}(t), T_{1}(t), T_{2}(t)\right\}$, and then for all $T>T_{3}$, the $T$-step Lyapunov drift becomes

$$
\begin{aligned}
V\left(\widetilde{\mathbf{z}}_{e}(t+T)\right)-V\left(\widetilde{\mathbf{z}}_{e}(t)\right) & \leq-\int_{t}^{t+T} a_{3}\left\|\widetilde{\mathbf{z}}_{e}(\tau)\right\|^{2} d \tau+a_{4} L \int_{t}^{t+T}\|u(\tau)\|\left\|\widetilde{\mathbf{z}}_{e}(\tau)\right\|^{2} d \tau \\
& \leq-\int_{t}^{t+T} a_{3}\left\|\widetilde{\mathbf{z}}_{e}(\tau)\right\|^{2} d \tau+a_{4} L\left(\frac{1}{T} \int_{t}^{t+T}\|u(\tau)\| d \tau \int_{t}^{t+T}\left\|\widetilde{\mathbf{z}}_{e}(\tau)\right\|^{2} d \tau+\delta T\right) \\
& \leq\left(-a_{3}+a_{4} L \cdot \frac{1}{T} \int_{t}^{t+T}\|u(\tau)\| d \tau\right) \int_{t}^{t+T}\left\|\widetilde{\mathbf{z}}_{e}(\tau)\right\|^{2} d \tau+a_{4} L \delta T \\
& \leq-a_{4} L \eta \int_{t}^{t+T}\left\|\widetilde{\mathbf{z}}_{e}(\tau)\right\|^{2} d \tau+a_{4} L \delta T \\
& \leq\left(-\eta \frac{\epsilon}{2}+\delta\right) a_{4} L T \\
& <0
\end{aligned}
$$

By the property of the Lyapunov function $V\left(\widetilde{\mathbf{z}}_{e}\right)$ in $(16)$, given any $t>0$, we have $\left\|\widetilde{\mathbf{z}}_{e}(t+T)\right\|<$ $\left\|\widetilde{\mathbf{z}}_{e}(t)\right\|$ for all $T>T_{3}$. Hence, $\lim _{t \rightarrow \infty}\left\|\widetilde{\mathbf{z}}_{e}(t)\right\|=0$, which contradicts to the assumption in (43). So the average trajectory $\overline{\left\|\widetilde{\mathbf{z}}_{e}\right\|^{2}}$ converges to 0 , and hence the tracking error $\overline{\left\|\widetilde{\mathbf{x}}_{e}\right\|^{2}}$ converges to 0 .

\section{REFERENCES}

[1] K. J. Arrow, L. Hurwicz, and H. Uzawa, Studies in linear and non-linear programming. California, Stanford: Stanford University Press, 1958.

[2] D. Feijer and F. Paganini, "Krasovskii's method in the stability of network control," in American Control Conference, ACC '09., Jun. 2009, pp. $3292-3297$.

[3] — "Stability of primal-dual gradient dynamics and applications to network optimization," Automatica, vol. 46, pp. 1974-1981, Dec. 2010. [Online]. Available: http://dx.doi.org/10.1016/j.automatica.2010.08.011]

[4] F. Kelly, A. Maulloo, and D. Tan, "Rate control for communication networks: Shadow prices, proportional fairness and stability," Journal of Operation Research, vol. 49, no. 3, pp. 237-252, 1998.

[5] J. Zhang, J. Zhang, S. Member, D. Zheng, and M. Chiang, "Impact of stochastic noisy feedback on distributed network utility maximization," IN INFOCOM 2007, vol. 2008, pp. 222-230, 2007.

[6] Y. Cheng and V. K. N. Lau, "Distributive power control algorithm for multicarrier interference network over time-varying fading channels: tracking performance analysis and optimization," IEEE Transations on Signal Processing, vol. 58, pp. 4750-4760, Sept. 2010. [Online]. Available: http://dx.doi.org/10.1109/TSP.2010.2052044

[7] X. Fan, T. Alpcan, M. Arcak, T. Wen, and T. Basar, "A passivity approach to game-theoretic cdma power control," Automatica, vol. 42, no. 11, pp. 1837 - 1847, 2006.

[8] T. Alpcan and T. Basar, "A hybrid systems model for power control in multicell wireless data networks," Performance Evaluation, vol. 57, no. 4, pp. 477 - 495, 2004.

[9] H. K. Khalil, Nonlinear Systems. Prentice-Hall, 1996.

[10] J. T. Wen, J. T. Wen, and M. Arcak, "A unifying passivity framework for network flow control," IEEE Transations on Automatic Control, vol. 49, pp. 162-174, 2002. 
[11] S. Boyd and L. Vandenberghe, Convex Optimization. Cambridge University Press, 2004.

[12] Y. Song and S. D. Blostein, "Mimo channel capacity in co-channel interference," in 21st Biennial Symposium on Communications, Kingston, Canada, Jan 2002, pp. 220-224.

[13] E. A. Jorswieck, H. Boche, and M. Weckerle, "Optimal transmitter and jamming strategies in gaussian mimo channels," in Vehicular Technology Conference, 2005. VTC 2005-Spring. 2005 IEEE 61st, vol. 2, 2005, pp. 978-982 Vol. 2. [Online]. Available: http://ieeexplore.ieee.org/xpls/abs_all.jsp?arnumber=1543452

[14] A. Kashyap, T. Basar, and R. Srikant, "Correlated jamming on mimo gaussian fading channels," IEEE Transations on Information Theory, vol. 50, no. 9, pp. 2119-2123, 2004.

[15] D. P. Palomar, D. P. Palomar, and M. Chiang, "Alternative distributed algorithms for network utility maximization: Framework and applications," IEEE Transations on Automatic Control, 2007.

[16] K. Baddour and N. Beaulieu, "Autoregressive models for fading channel simulation," in Global Telecommunications Conference, 2001. IEEE GLOBECOM '01., vol. 2, 2001, pp. 1187 -1192 vol.2.

[17] M. Tsatsanis and Z. Xu, "Pilot symbol assisted modulation in frequency selective fading wireless channels," IEEE Transactions on Signal Processing, vol. 48, no. 8, pp. 2353 -2365, Aug 2000.

[18] P.-Y. Wu and A. Duel-Hallen, "Multiuser detectors with disjoint kalman channel estimators for synchronous cdma mobile radio channels," IEEE Transactions on Communications, vol. 48, no. 5, pp. 752 -756, May 2000.

[19] H.-Y. Wu and A. Duel-Hallen, "On the performance of coherent and noncoherent multiuser detectors for mobile radio cdma channels," in 5th IEEE International Conference on Universal Personal Communications, 1996, vol. 1, Oct 1996, pp. $76-80$ vol.1.

[20] A. Nedić and A. Ozdaglar, "Subgradient methods for saddle-point problems," Journal of Optimization Theory and Applications, vol. 142, pp. 205-228, 2009.

[21] M. Kallio, M. Kallio, M. Kallio, C. H. Rosa, and C. H. Rosa, "Large-scale convex optimization via saddle point computation," Operations Research, vol. 47, pp. 93-101, 1994.

[22] T. ElBatt and A. Ephremides, "Joint scheduling and power control for wireless ad hoc networks," IEEE Transactions on Wireless Communications, vol. 3, no. 1, pp. 74 - 85, Jan 2004.

[23] M. Kallio and A. Ruszczynski, "Perturbation methods for saddle point computation," International Institute for Applied Systems Analysis, Working Papers, 1994. [Online]. Available: http://econpapers.repec.org/RePEc:wop:iasawp:wp94038 


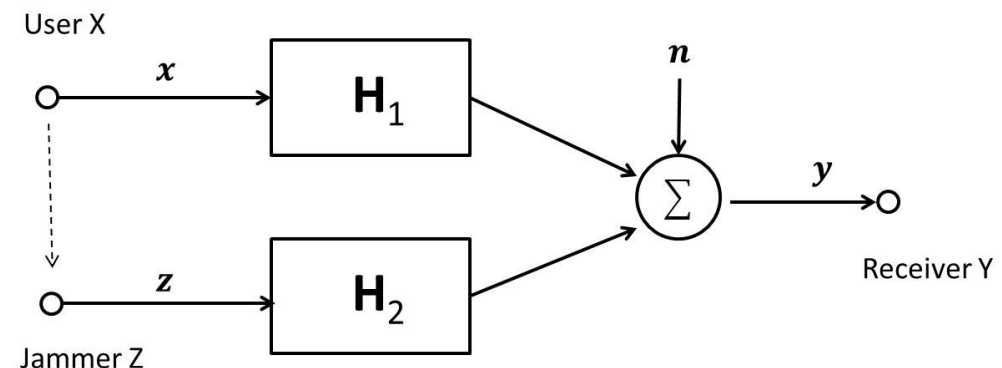

Figure 1. A system model for a point-to-point MIMO with a jammer. The user $\mathrm{X}$ transmits signal to $\mathrm{Y}$ through the channel $\mathbf{H}_{1}$ and a jammer $\mathrm{Z}$ transmits a jamming signal to $\mathrm{Y}$ through the channel $\mathbf{H}_{2}$.

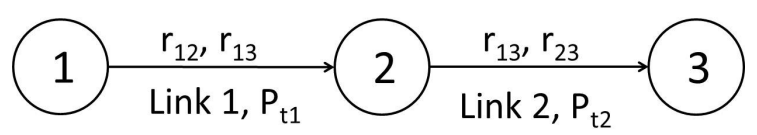

Figure 2. A specific example of the multihop wireless network with 3 nodes and 2 links, where the collection of traffic flows is given by $\mathcal{C}=\{(1,2),(1,3),(2,3)\}$, and the sets of links are $L(1,2)=\{1\}, L(1,3)=\{1,2\}$ and $L(2,3)=\{2\}$. $P_{t 1}$ and $P_{t 2}$ denote the total power allocated to link 1 and link 2 respectively.

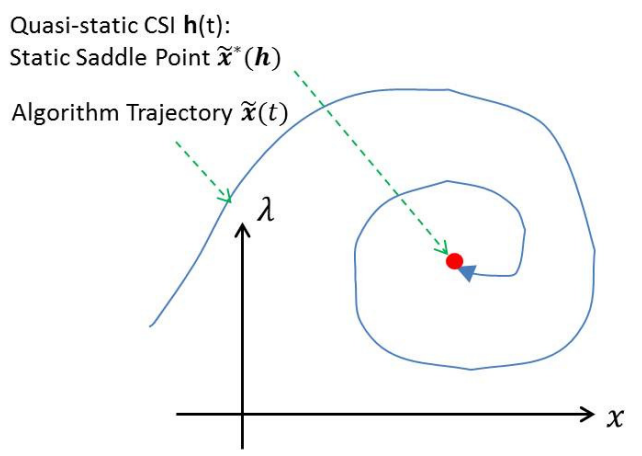

(a)

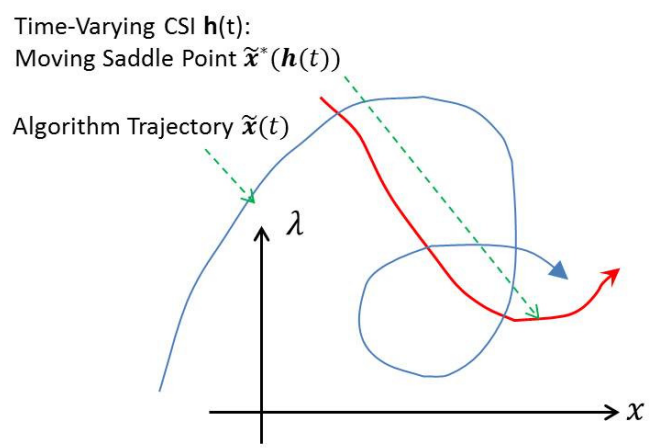

(b)

Figure 3. An illustration of the convergence behavior of the primal-dual algorithms. In (a), the equilibrium point in the equivalent virtual dynamic system is asymptotically stable when the CSI $\mathbf{h}(t)$ is quasi-static [1]. However, in (b), when the CSI $\mathbf{h}(t)$ is time-varying, the equilibrium point $\widetilde{\mathbf{x}}^{*}(t)$ becomes also time-varying and the convergence is not guaranteed. 


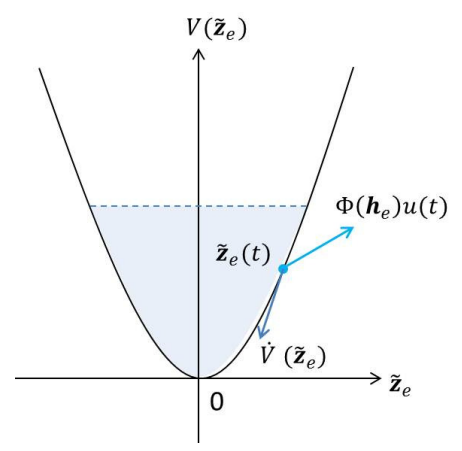

Figure 4. An illustration of the excitation due to the exogenous input $\Phi\left(\mathbf{h}_{e}\right) u(t)$ for the equivalent virtual dynamic system $\widetilde{\mathcal{Z}}_{e}$. We can visualize the Lyapunov function $V\left(\widetilde{\mathbf{z}}_{e}\right)$ as an energy function. Without the time-varying channel effect $\left(u(t)=0\right.$ ), the algorithm trajectory will eventually converges to the equilibrium $\widetilde{\mathbf{z}}_{e}^{*}=0$ (corresponding to exponential convergence of the primal-dual iterations in (2)-(3) because there is no exogenous excitation energy applied to the system. With the time-varying channels, there is an uncertainty region (shaded region) where the system state will converge to (corresponds to the convergence region $\widetilde{D}_{e}$ in Corollary 1 and $D_{e}$ in Corollary 2). The region is bounded and the size depends on the "energy" applied to the system due to the exogenous input $\Phi\left(\mathbf{h}_{e}\right) u(t)$.

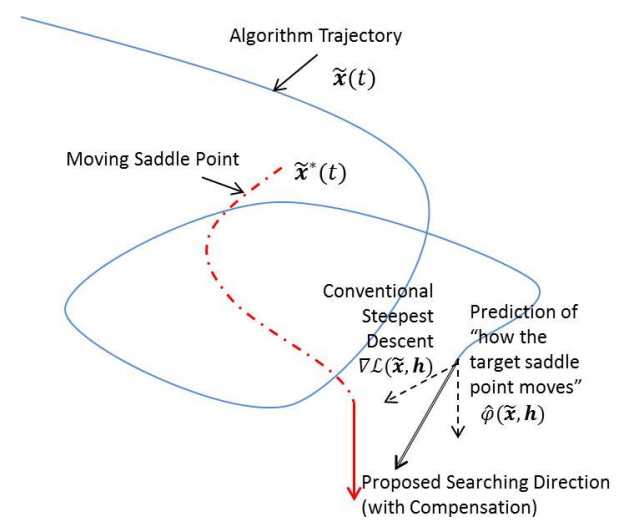

Figure 5. An illustration of the proposed algorithm with adaptive compensation to improve the convergence behavior of the primal-dual iterations in time-varying channels. Instead of searching via the steepest descent direction $\nabla \mathcal{L}(\widetilde{\mathbf{x}}, \mathbf{h})$ in the conventional primal-dual algorithm, it searches in a compensated direction $\nabla \mathcal{L}(\widetilde{\mathbf{x}}, \mathbf{h})+\widehat{\varphi}(\widetilde{\mathbf{x}}, \mathbf{h})$ to offset the potential movement of the saddle point $\widetilde{\mathbf{x}}^{*}(t)$.

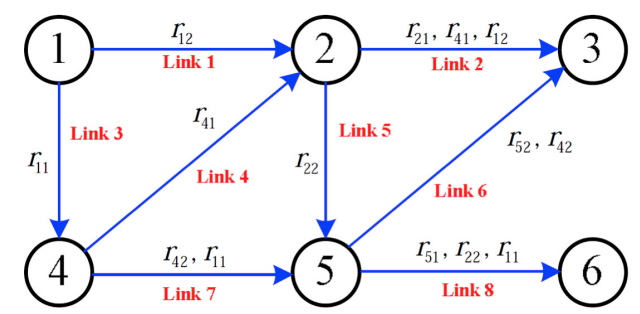

Figure 6. A specific example of wireless ad hoc network with 6 nodes 8 directed links and 8 data flows. The data flows are delivered simutaneously with fixed routes. Links from the same transmitting node occupy different subbands and do not interfere with each other. The interference at each receiving node is handled by multiuser detection (MUD) techniques. Allowable transmission rate for each link is determined by the capacity region at the corresponding receiving node. 


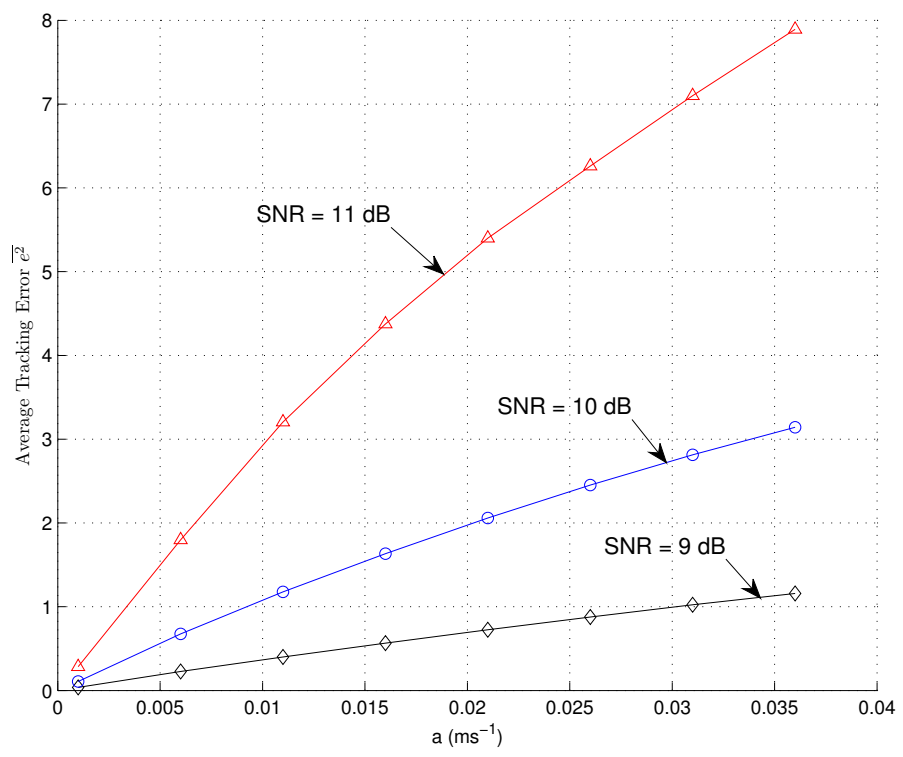

Figure 7. The convergence performance of the primal-dual algorithm for the strongly concave-convex saddle point problem given in Section III-B The CSI model is given by $\dot{\mathbf{h}}=A(\mathbf{h}-\overline{\mathbf{h}})+\sqrt{2 a} w(t)$, where $A=-a I, \overline{\mathbf{h}}=\mathbf{1}$, and $w(t)$ is a zero-mean unit-variance white Gaussian process. The variance of the CSI $\mathbf{h}(t)$ is normalized to unity, and the parameter $a$ controls the time-correlation of the CSI. It shows that as the SNR increases, the tracking error also increases for the same CSI variation rate in terms of $a$.

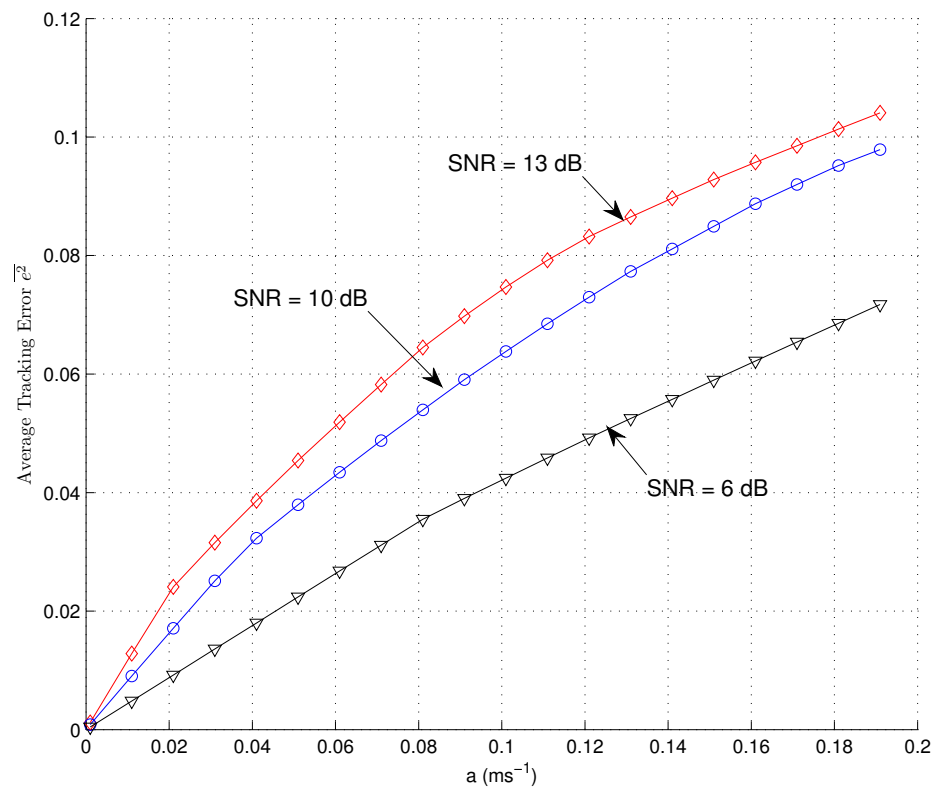

Figure 8. The convergence performance of the primal-dual algorithm for the degraded saddle point problem given in Section IV-B The CSI model is given by $\dot{\mathbf{h}}=-a(\mathbf{h}-\overline{\mathbf{h}})+\sqrt{2 a} w(t)$, where $\overline{\mathbf{h}}=\mathbf{1}$ and $w(t)$ is a zero-mean unit-variance white Gaussian process. The variance of the CSI $\mathbf{h}(t)$ is normalized to unity, and the parameter $a$ controls the time-correlation of the CSI. It shows that as the SNR increases, the tracking error also increases for the same CSI variation rate in terms of $a$. 


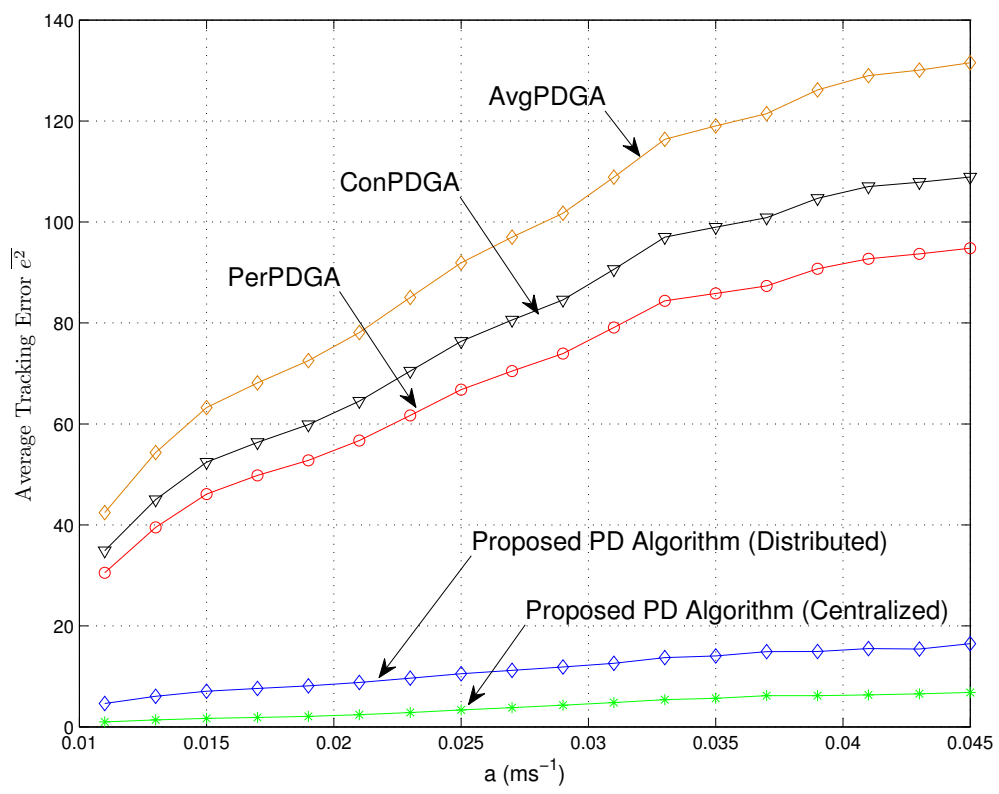

Figure 9. The convergence performance comparison of the proposed algorithms and the baseline algorithms [3], [21], [20], which solve a NUM under the wireless network in Fig 6 The CSI model is given by $\dot{\mathbf{h}}=-a(\mathbf{h}-\overline{\mathbf{h}})+\sqrt{2 a} w(t)$, where $\overline{\mathbf{h}}=\mathbf{1}$ and $w(t)$ is a zero-mean unit-variance white Gaussian process. The variance of the CSI $\mathbf{h}(t)$ is normalized to unity, and the parameter $a$ controls the time-correlation of the CSI. The tracking errors of the proposed algorithms are much smaller than those of the baselines.

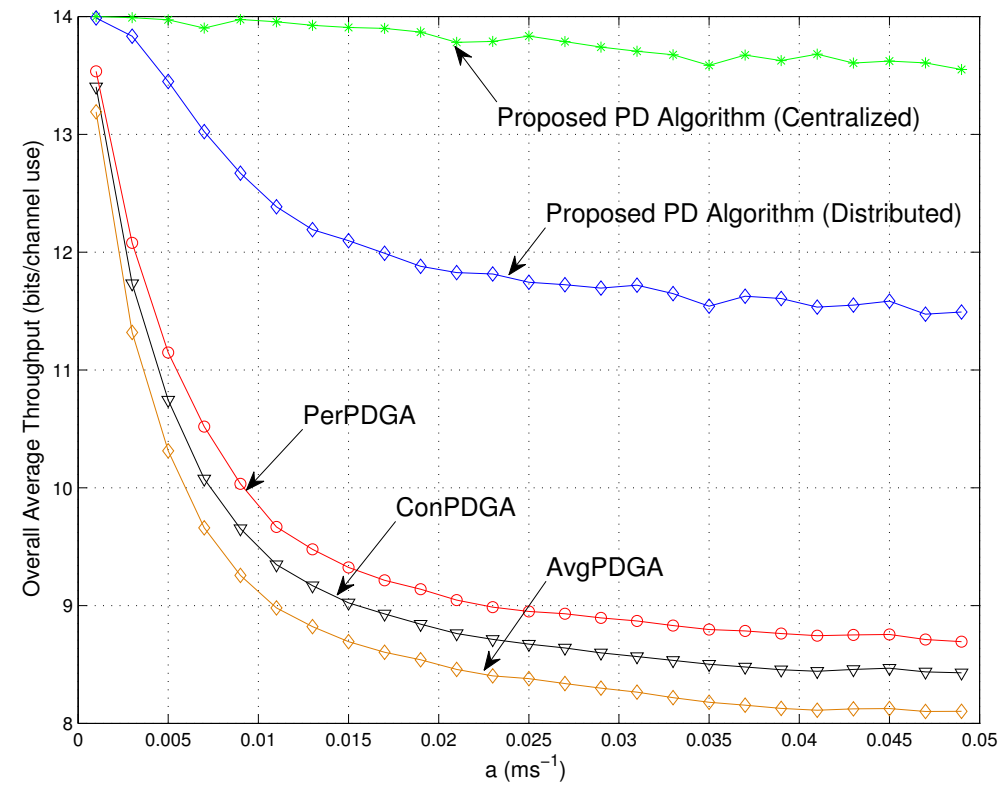

Figure 10. Average network throughput versus the channel fading rate parameter $a$, while applying the various proposed and baseline algorithms to solve the NUM problem under the network in Fig 6 The average throughput decreases when the fading rate $a$ increases. The proposed algorithms have much higher network throughput than all the other baselines. 